\title{
Macroscopic Maxwell's equations and negative index materials
}

\author{
B. Gralak ${ }^{1}$ and A. Tip ${ }^{2, a)}$ \\ ${ }^{1}$ Institut Fresnel, CNRS, Aix-Marseille Université, Ecole Centrale Marseille, Campus de St \\ Jérôme, 13397 Marseille Cedex 20, France \\ ${ }^{2}$ FOM-Instituut AMOLF, Science Park 104, 1098 XG Amsterdam, The Netherlands
}

(Received 4 December 2009; accepted 9 March 2010; published online 13 May 2010)

\begin{abstract}
We study the linear phenomenological Maxwell's equations in the presence of a polarizable and magnetizable medium (magnetodielectric). For a dispersive, nonabsorptive, medium with equal electric and magnetic permeabilities $\varepsilon(\omega)$ and $\mu(\omega)$, the latter can assume the value of $-1(+1$ is their vacuum value) for a discrete set of frequencies $\pm \hat{\omega}_{n}$, i.e., for these frequencies the medium behaves as a negative index material (NIM). We show that such systems have a well-defined time evolution. In particular, the fields remain square integrable (and the electromagnetic energy finite) if this is the case at some initial time. Next we turn to Green's function $G(\mathbf{x}, \mathbf{y}, z)$ (a tensor), associated with the electric Helmholtz operator for a set of parallel layers filled with a material. We express it in terms of the well-known scalar $s$ and $p$ ones. For a half space filled with the material and with a single dispersive Lorentz form for $\varepsilon(\omega)=\mu(\omega)$, we obtain an explicit form for G. We find the usual behavior for NIMs for $\omega= \pm \hat{\omega}$, there is no refection outside the evanescent regime and the transmission (refraction) shows the usual NIM behavior. We find that $G$ has poles in $\pm \hat{\omega}$, which lead to a modulation of the radiative decay probability of an excited atom. The formalism is free from ambiguities in the sign of the refractive index. (c) 2010 American Institute of Physics.
\end{abstract}

[doi:10.1063/1.3374670]

\section{INTRODUCTION}

Often magnetization plays a minor role in situations where the phenomenological Maxwell's equations apply. But in recent years negative index materials (NIMs), also called left handed materials, have become of increasing interest, in particular, due to the work of Veselago ${ }^{1}$ and Pendry. ${ }^{2}$ Here the magnetization is not negligible at all. In general, a NIM system is defined by the property that for certain frequencies $\omega$ the electric permeability (permittivity) $\varepsilon(\omega)$ or the magnetic permeability $\mu(\omega)$ becomes negative. Of particular interest is the case where both become negative at the same frequency $\hat{\omega}$, the NIM frequency, and are equal to the opposite of their vacuum value, i.e., -1 instead of +1 . Below we refer to this case as the NIM situation.

For an introduction, containing an extensive set of references, see Ref. 3 (there are also NIM systems based on specific properties of photonic crystals, which are not considered here).

The existence of NIMs has been debated in the theoretical literature at various occasions. ${ }^{4}$ In particular, the sign of the index of refraction, which involves taking a square root, has been a subject of discussion. Naively it equals +1 , in both vacuum and a NIM system but this result is challenged for the NIM situation. For experimental verification, see Ref. 5. Calculations based upon a simple model, where one part of space is vacuum $(\varepsilon=\mu=1)$ and the other filled with a NIM

\footnotetext{
${ }^{a)}$ Electronic mail: tip@amolf.nl.
} 
( $\varepsilon=\mu=-1$, frequency independent) tend to give ambiguous results. This is sometimes remedied by adding a small imaginary part to one of the permeabilities but on the whole the situation is rather unclear.

The use of the phenomenological Maxwell's equations should solve possible ambiguities but it seems that so far this approach has not been taken and here we intend to fill this gap. Since fabricated materials, intended to study NIM behavior, are usually anisotropic, we take the (space and frequency dependent) susceptibilities (which relate the polarization and magnetization to the electric and magnetic fields) to be tensors rather than scalars.

The first matter to be solved is the existence of a proper time evolution. In view of the time convolutions in the constitutive equations this is not directly obvious. The next task is to see if a NIM situation can exist. This being the case, the following point of interest is obtaining the Helmholtz Green's function and scattering amplitudes for specific configurations. The former is important since its imaginary part enters the radiative decay rate of an atom or nanostructure close to the material. Hence experimental results on such decay rates can give information about the properties of the material. In addition Green's function, or rather the associated transition operator, is required to describe scattering phenomena, such as reflection and transmission in layered systems.

Thus we start with the phenomenological Maxwell's equations with general frequencydependent permeability tensors, satisfying the usual causality and passivity conditions. After providing some relevant background and a summary of the properties of the electric and magnetic susceptibilities we introduce the auxiliary field formalism (AFF). The latter was presented earlier by one of us (Tip in Ref. 6) for dielectrics $(\mu=1)$. The idea is to introduce an additional set of fields, the auxiliary fields, to remove the time convolutions in Maxwell's equations. This has a number of advantages.

(1) The combined set of electromagnetic and auxiliary fields satisfies a unitary time evolution, thus insuring a proper time evolution for the electromagnetic fields.

(2) Such a system is easily quantized, leading to a second quantization formalism that is rigorously valid for both absorptive and dispersive systems.

(3) The formalism implies that the inverses of the electric and magnetic Helmholtz operators exist as bounded operators, so the associated Green's functions are square integrable.

(4) Setting up a scattering formalism is straightforward.

The AFF leads to a proper time evolution, notwithstanding the possibility that for specific frequencies $\hat{\omega}$ we can have a NIM situation, $\varepsilon(\hat{\omega})=\mu(\hat{\omega})=-1$. In case the initial fields are square integrable they remain so for all later times. In Appendices A and $\mathrm{C}$ we give a rigorous proof of this important fundamental property.

Another relevant piece of information is that the susceptibilities for general dispersive, nonabsorptive, systems consist of a (possibly infinite) sum of Lorentz contributions (Tip in Ref. 7). This immediately gives a positive answer to the existence of NIMs. In case we are dealing with a single dispersive Lorentz contribution,

$$
\varepsilon(\omega)=\mu(\omega)=1-\frac{\Omega^{2}}{\omega^{2}-\omega_{0}^{2}},
$$

we note that for the NIM frequencies $\omega= \pm \hat{\omega}, \hat{\omega}^{2}=\omega_{0}^{2}+\Omega^{2} / 2$ we have $\varepsilon( \pm \hat{\omega})=\mu( \pm \hat{\omega})=-1$. Thus, theoretically, the NIM case can be realized for dispersive, nonabsorptive, systems, contrary to what is sometimes claimed. ${ }^{4}$ Adding more Lorentz terms gives more frequency values with this property but it remains a discrete set and in between the values of $\varepsilon(\omega)$ and $\mu(\omega)$ vary wildly. In fact, between two subsequent NIM frequencies there is always a frequency for which total reflection takes place (for $\omega=\omega_{0}$ in the above example), precisely the opposite of the NIM case, where no reflection is thought to be the situation. Thus it seems that obtaining an extended frequency interval for which the permeabilities are approximately equal to -1 is not possible. In fact, it was already noted by Veselago ${ }^{1}$ that a system showing NIM behavior must be dispersive. In case there 
is absorption, $\varepsilon(\omega)=\mu(\omega)=-1$ cannot be realized for real $\omega$ as can be seen by adding absorption to the above case,

$$
\varepsilon(\omega)=\mu(\omega)=1-\frac{\Omega^{2}}{\omega^{2}+i \gamma \omega-\omega_{0}^{2}} .
$$

Next we introduce the Laplace-transformed Maxwell's equations and the tensor Green's function $\mathrm{G}(\mathbf{x}, \mathbf{y}, z)$ related to the electric Helmholtz operator. $\mathrm{G}(\mathbf{x}, \mathbf{y}, z)$ features most of the properties of the system. As said the radiative decay rate of excited atoms is proportional to its imaginary part. We then turn to layered systems and express $\mathrm{G}(\mathbf{x}, \mathbf{y}, z)$ into a set of two scalar ones for $s$ and $p$ polarization, respectively. Subsequently Green's function for the half space case, mentioned above, is studied. In particular, we obtain an explicit expression for $\mathbf{G}(\mathbf{x}, \mathbf{y}, z)$. Then $\boldsymbol{E}(\mathbf{x}, t)$ is given by the inverse Laplace transform of

$$
\hat{\boldsymbol{E}}(\mathbf{x}, z)=\int d \mathbf{y G}(\mathbf{x}, \mathbf{y}, z) \cdot \mathbf{g}(\mathbf{y}, z),
$$

where $\mathbf{g}(\mathbf{y}, z)$ is some square integrable initial field configuration or an external current density. At this point the square root of $z^{2} \varepsilon(\mathbf{x}, z) \mu(\mathbf{x}, z)-\kappa^{2}$, with $\boldsymbol{\kappa}$ a two-dimensional wave vector, must be evaluated as $\delta>0$ in $z=\omega+i \delta$ tends to 0 . Depending on the values of $\mathbf{x}$ and $\omega$ different results are obtained, it can be positive, negative, or imaginary. We find that reflective contributions to $\mathrm{G}(\mathbf{x}, \mathbf{y}, \pm \hat{\omega}+i 0)$ vanish in the radiative regime, $\hat{\omega}>\kappa$, and transmission is also modified substantially. This confirms the results by Pendry. ${ }^{2}$ In the evanescent regime $\mathbf{G}(\mathbf{x}, \mathbf{y}, z)$ has poles in $z$ $= \pm \hat{\omega}$, giving finite contributions, proportional to $\exp [ \pm i \hat{\omega} t]$, to the electric field $\boldsymbol{E}(\mathbf{x}, t)$. It turns out that $\mathrm{K}$, the generator of the time evolution in the AFF, has $\pm \hat{\omega}$ as eigenvalues with infinite degeneracy, the latter giving rise to the above poles. Although we do not discuss quantization, we note that this feature gives rise to an interesting structure of the associated field Hamiltonian. In addition to the eigenvalue 0 , associated with the vacuum state, now $\pm \hat{\omega}$ are also eigenvalues. This affects radiative decay constants of excited atoms, as is discussed in Sec. VII.

A word about notation: With a dispersive system we mean a dispersive, nonabsorptive system. Inner products are denoted as $(f, g)=\langle g \mid f\rangle$. The unit vector along $\mathbf{a} \in \mathbb{R}^{3}$ is $\mathbf{e}_{\mathbf{a}}=\mathbf{a} / a, a=|\mathbf{a}|$. The three Cartesian axes are denoted by $X_{1}, X_{2}$, and $X_{3}$ with corresponding unit vectors $\mathbf{e}_{1}, \mathbf{e}_{2}$, and $\mathbf{e}_{3}$. The component of $\mathbf{a} \perp \mathbf{e}_{3}$ is denoted by $\mathbf{a}^{\perp}$. $U$ is the unit $3 \times 3$ matrix. Transposes of matrices are indicated by means of the superscript $T$ and their Hermitean adjoints by $\dagger$. Square roots are defined in the usual way with non-negative imaginary part. $\mathrm{I}_{\mathcal{A}}(x)$ is the characteristic function for the set $\mathcal{A}, \mathrm{I}_{\mathcal{A}}(x)=1$ for $x \in \mathcal{A}$ and $\mathrm{I}_{\mathcal{A}}(x)=0$ for $x \notin \mathcal{A}$.

\section{BACKGROUND}

Starting point is the set of linear phenomenological Maxwell's equations for the case that permanent polarization and magnetization are absent (we set $\varepsilon_{0}=\mu_{0}=1$ for brevity),

$$
\begin{gathered}
\partial_{t} \boldsymbol{D}(\mathbf{x}, t)=\partial_{\mathbf{x}} \times \boldsymbol{H}(\mathbf{x}, t), \quad \partial_{t} \boldsymbol{B}(\mathbf{x}, t)=-\partial_{\mathbf{x}} \times \boldsymbol{E}(\mathbf{x}, t), \\
\partial_{\mathbf{x}} \cdot \boldsymbol{D}(\mathbf{x}, t)=0, \quad \partial_{\mathbf{x}} \cdot \boldsymbol{B}(\mathbf{x}, t)=0,
\end{gathered}
$$

with the constitutive equations

$$
\boldsymbol{D}(\mathbf{x}, t)=\boldsymbol{E}(\mathbf{x}, t)+\boldsymbol{P}(\mathbf{x}, t), \quad \boldsymbol{P}(\mathbf{x}, t)=\int_{t_{0}}^{t} d s \boldsymbol{\chi}_{e}(\mathbf{x}, t-s) \cdot \boldsymbol{E}(\mathbf{x}, s),
$$




$$
\boldsymbol{H}(\mathbf{x}, t)=\boldsymbol{B}(\mathbf{x}, t)-\boldsymbol{M}(\mathbf{x}, t), \boldsymbol{M}(\mathbf{x}, t)=\int_{t_{0}}^{t} d s \boldsymbol{\chi}_{m}(\mathbf{x}, t-s) \cdot \boldsymbol{H}(\mathbf{x}, s) .
$$

Here $\boldsymbol{\chi}_{e}(\mathbf{x}, t)$ and $\boldsymbol{\chi}_{m}(\mathbf{x}, t)$ are the electric and magnetic susceptibility tensors. We also introduce the current densities $\boldsymbol{J}_{e}(\mathbf{x}, t)=\partial_{t} \boldsymbol{P}(\mathbf{x}, t)$ and $\boldsymbol{J}_{m}(\mathbf{x}, t)=\partial_{t} \boldsymbol{M}(\mathbf{x}, t)$. Causality requires that the susceptibilities $\boldsymbol{\chi}_{e}(\mathbf{x}, t)$ and $\boldsymbol{\chi}_{m}(\mathbf{x}, t)$ vanish for $t<0$. Assuming no initial surges in $\boldsymbol{P}(\mathbf{x}, t)$ and $\boldsymbol{M}(\mathbf{x}, t)$ at $t=t_{0}$, so $\boldsymbol{J}_{e}\left(\mathbf{x}, t_{0}\right)=\boldsymbol{J}_{m}\left(\mathbf{x}, t_{0}\right)=0$, we also have $\boldsymbol{\chi}_{e}\left(\mathbf{x}, t_{0}\right)=\boldsymbol{\chi}_{m}\left(\mathbf{x}, t_{0}\right)=0$. Indeed, currents are due to the motion of charged, massive, particles and their velocity cannot be changed instantaneously. This property is found in linear response expressions and also, for instance, for the Lorentz case. Denoting $\partial_{t} \boldsymbol{\chi}(t)=\boldsymbol{\chi}^{\prime}(t)$, we then obtain

$$
\boldsymbol{J}_{e}(\mathbf{x}, t)=\int_{t_{0}}^{t} d s \boldsymbol{\chi}_{e}^{\prime}(\mathbf{x}, t-s) \cdot \boldsymbol{E}(\mathbf{x}, s), \boldsymbol{J}_{m}(\mathbf{x}, t)=\int_{t_{0}}^{t} d s \boldsymbol{\chi}_{m}^{\prime}(\mathbf{x}, t-s) \cdot \boldsymbol{H}(\mathbf{x}, s) .
$$

As a matrix, $\boldsymbol{\chi}_{e, m}(\mathbf{x}, t)$ are assumed to be symmetric (this property explicitly holds for linear response expressions if the unperturbed matter Hamiltonian is time-reversal invariant).

\section{Remarks:}

(1) The initial time $t_{0}$ can have any value, in particular, $t_{0}=-\infty$. However, in view of the Laplacetransformed equations, introduced later on, $t_{0}=0$ is a convenient choice. This case is often realized in practical situations. For instance, in describing scattering of an electromagnetic wave packet with bounded support from a material object, the wave packet is initially, as $t \rightarrow-\infty$, well separated from the object and, in view of the hyperbolic nature of Maxwell's equations, the support remains bounded and, contrary to the Schrödinger case, it takes a nonzero time for the support to reach the object. Thus the polarization and magnetization vanish for times smaller than some finite $t_{0}$, which we set equal to 0 .

(2) It is customary ${ }^{8}$ to relate $\boldsymbol{M}$ to $\boldsymbol{H}$, rather than $\boldsymbol{B}$, although $\boldsymbol{B}$ is the more fundamental field. Indeed, interactions with atoms are in terms of the microscopic vector potential $\boldsymbol{A}$, which is related to the microscopic $\boldsymbol{B}$-field. But note that if the particles are in vacuum, sufficiently far away from the medium, the microscopic $\boldsymbol{B}$-field equals the macroscopic one and both equal $\boldsymbol{H}$ at the particle coordinates. We note further that linear response expressions usually relate the magnetization to the (microscopic) $\boldsymbol{B}$-field.

Next we introduce the Fourier decomposition,

$$
\begin{gathered}
\boldsymbol{\chi}_{e, m}^{\prime}(\mathbf{x}, t)=\int d \lambda \exp [-i \lambda t] \boldsymbol{\nu}_{e, m}(\mathbf{x}, \lambda), \\
\boldsymbol{\nu}_{e, m}(\mathbf{x}, \lambda)=\frac{1}{2 \pi} \int d t \exp [i \lambda t] \boldsymbol{\chi}_{e, m}^{\prime}(\mathbf{x}, t)=\frac{1}{2 \pi} \int_{0}^{\infty} d t \exp [i \lambda t] \boldsymbol{\chi}_{e, m}^{\prime}(\mathbf{x}, t) .
\end{gathered}
$$

Since $\boldsymbol{\chi}_{e, m}^{\prime}(\mathbf{x}, t)$ are real, we have $\boldsymbol{\nu}_{e, m}(\mathbf{x},-\lambda)=\overline{\boldsymbol{\nu}_{e, m}(\mathbf{x}, \lambda)}$ in the sense that this relation holds for each component of these tensors. We also assume that the system is passive. This means that the electromagnetic energy,

$$
\mathcal{E}_{e m}(t)=\frac{1}{2} \int d \mathbf{x}\left\{\boldsymbol{E}(\mathbf{x}, t)^{2}+\boldsymbol{H}(\mathbf{x}, t)^{2}\right\}
$$

cannot increase as a function of time. So initial population inversions in the material system are excluded. Then 


$$
\begin{aligned}
\mathcal{E}_{e m}(t)-\mathcal{E}_{e m}\left(t_{0}\right)= & \int_{t_{0}}^{t} d s \partial_{s} \mathcal{E}_{e m}(s) \\
= & -\int_{t_{0}}^{t} d s \int d \mathbf{x}\left\{\boldsymbol{J}_{e}(\mathbf{x}, s) \cdot \boldsymbol{E}(\mathbf{x}, s)+\boldsymbol{J}_{m}(\mathbf{x}, s) \cdot \boldsymbol{H}(\mathbf{x}, s)\right\} \\
= & -\int_{t_{0}}^{t} d s \int_{t_{0}}^{s} d u \int d \mathbf{x}\left\{\boldsymbol{\chi}_{e}^{\prime}(\mathbf{x}, s-u): \boldsymbol{E}(\mathbf{x}, u) \boldsymbol{E}(\mathbf{x}, s)+\boldsymbol{\chi}_{m}^{\prime}(\mathbf{x}, s-u): \boldsymbol{H}(\mathbf{x}, u) \boldsymbol{H}(\mathbf{x}, s)\right\} \\
= & -\int_{t_{0}}^{t} d s \int_{t_{0}}^{t} d u \int d \mathbf{x}\left\{\boldsymbol{\chi}_{e}^{\prime}(\mathbf{x}, s-u): \boldsymbol{E}(\mathbf{x}, u) \boldsymbol{E}(\mathbf{x}, s)+\boldsymbol{\chi}_{m}^{\prime}(\mathbf{x}, s-u): \boldsymbol{H}(\mathbf{x}, u) \boldsymbol{H}(\mathbf{x}, s)\right\} \\
= & -\int d \mathbf{x}\left[\boldsymbol{\nu}_{e}(\mathbf{x}, \lambda):\left\{\int_{t_{0}}^{t} d u \exp [i \lambda u] \boldsymbol{E}(\mathbf{x}, u)\right\}\left\{\int_{t_{0}}^{t} d s \exp [-i \lambda s] \boldsymbol{E}(\mathbf{x}, s)\right\}\right. \\
& +\boldsymbol{\nu}_{m}(\mathbf{x}, \lambda):\left\{\int_{t_{0}}^{t} d u \exp [i \lambda u] \boldsymbol{H}(\mathbf{x}, u)\right\}\left\{\int_{t_{0}}^{t} d s \exp [-i \lambda s] \boldsymbol{H}(\mathbf{x}, s)\right\} \leqq 0,
\end{aligned}
$$

So

$$
\boldsymbol{\nu}_{e, m}(\mathbf{x}, \lambda) \geq 0 .
$$

This result also emerges in linear response expressions if the initial density operator for the material system is a function of its Hamiltonian and the level population decreases with increasing energy as is the case for a canonical distribution. Now $\boldsymbol{\nu}_{e, m}(\mathbf{x},-\lambda)=\boldsymbol{\nu}_{e, m}(\mathbf{x}, \lambda) \geq 0$, leading to

$$
\begin{gathered}
\boldsymbol{\chi}_{e}(\mathbf{x}, t)=\int d \lambda \frac{\sin (\lambda t)}{\lambda} \boldsymbol{\nu}_{e}(\mathbf{x}, \lambda), \boldsymbol{\chi}_{m}(\mathbf{x}, t)=\int d \lambda \frac{\sin (\lambda t)}{\lambda} \boldsymbol{\nu}_{m}(\mathbf{x}, \lambda), \\
\boldsymbol{\chi}_{e}^{\prime}(\mathbf{x}, t)=\int d \lambda \cos (\lambda t) \boldsymbol{\nu}_{e}(\mathbf{x}, \lambda), \boldsymbol{\chi}_{m}^{\prime}(\mathbf{x}, t)=\int d \lambda \cos (\lambda t) \boldsymbol{\nu}_{m}(\mathbf{x}, \lambda) .
\end{gathered}
$$

We introduce Laplace transforms according to $(\operatorname{Im} z>0)$,

$$
\hat{f}(z)=\int_{0}^{\infty} d t \exp [i z t] f(t), f(t)=\frac{1}{2 \pi} \int_{\Gamma} d z \exp [-i z t] \hat{f}(z),
$$

where $\Gamma$ is a path running from $-\infty$ to $+\infty$ at some distance $\delta>0$ parallel to the real axis. Then, for $t_{0} \leq 0$, performing a partial integration and using $\boldsymbol{\chi}_{e, m}(t)=0, t<0$,

$$
\begin{aligned}
\hat{\boldsymbol{\chi}}_{e, m}(\mathbf{x}, z) & =\int_{t_{0}}^{\infty} d t \exp [i z t] \boldsymbol{\chi}_{e, m}(\mathbf{x}, t) \\
& =\int_{0}^{\infty} d t \exp [i z t] \boldsymbol{\chi}_{e, m}(\mathbf{x}, t) \\
& =-\frac{1}{i z} \int_{0}^{\infty} d t \exp [i z t] \boldsymbol{\chi}_{e, m}^{\prime}(\mathbf{x}, t) \\
& =-\frac{1}{i z} \int_{0}^{\infty} d t \exp [i z t] \int d \lambda \boldsymbol{\nu}_{e, m}(\mathbf{x}, \lambda) \exp [-i \lambda t] \\
& =\int d \lambda \boldsymbol{\nu}_{e, m}(\mathbf{x}, \lambda) \frac{1}{\lambda^{2}-z^{2}} .
\end{aligned}
$$


Causality and passivity imply that these are the most general expressions for $\hat{\boldsymbol{\chi}}_{e, m}(\mathbf{x}, z)$ (Ref. 7) (the latter, being analytic in the upper half plane, are the so-called Herglotz functions, which can always be represented in the above form). In general, $\boldsymbol{\nu}_{e, m}(\mathbf{x}, \lambda)$ is made up of integrable functions, leading to absorptive systems, and of $\delta$-function contributions. The second give rise to a set of dispersive Lorentz terms. In fact, we can say that causal, passive, dispersive susceptibilities consist of a (possibly infinite) sum of dispersive Lorentz terms. ${ }^{7}$ Then, in the isotropic and dispersive case, $\hat{\boldsymbol{\chi}}(\mathbf{x}, z)=\hat{\chi}(\mathbf{x}, z) \cup$,

$$
\nu_{e, m}(\mathbf{x}, \lambda)=\sum_{n} \Omega_{e, m}^{n}(\mathbf{x})^{2} \delta\left(\lambda-\lambda_{e, m}^{n}\right), \quad \hat{\chi}_{e, m}(\mathbf{x}, z)=-\sum_{n} \frac{\Omega_{e, m}^{n}(\mathbf{x})^{2}}{z^{2}-\left(\lambda_{e, m}^{n}\right)^{2}} .
$$

Here we recall that, disregarding $\mathbf{x}$-dependencies, an absorptive Lorentz contribution is given by

$$
\hat{\chi}_{\gamma}(z)=-\frac{\Omega^{2}}{z^{2}+i \gamma z-\omega_{0}^{2}}, \quad \chi_{\gamma}(t)=\Omega^{2} \exp \left[-\frac{\gamma t}{2}\right] \frac{\sin \omega_{1} t}{\omega_{1}}, \omega_{1}=\sqrt{\omega_{0}^{2}+\frac{\gamma^{2}}{4}}, \Omega, \omega_{0}, \gamma>0 .
$$

For $\omega \in \mathbb{R}$,

$$
\begin{gathered}
\hat{\chi}_{e, m}(\mathbf{x}, \omega)=\int_{0}^{\infty} d t \exp [i \omega t] \chi_{e, m}(t)=\frac{1}{\omega} \int d \lambda \frac{1}{\lambda-\omega-i 0} \nu_{e, m}(\mathbf{x}, \lambda), \\
\operatorname{Im} \hat{\chi}_{e, m}(\mathbf{x}, \omega)=\frac{\pi}{\omega} \nu_{e, m}(\mathbf{x}, \omega) .
\end{gathered}
$$

In the dispersive case, $\gamma=0$,

$$
\hat{\chi}(\omega+i 0)=-\frac{\Omega^{2}}{\omega^{2}-\omega_{0}^{2}}+i \pi \delta\left(\omega^{2}-\omega_{0}^{2}\right), \quad \chi(t)=\Omega^{2} \frac{\sin \omega_{0} t}{\omega_{0}} .
$$

Here the $\delta$-function insures the validity of the Kramers-Kronig relations although it does not play a further role. ${ }^{7}$ Negative index systems are characterized by negative real permeabilities for some real frequencies. In the Lorentz case this can happen at a few discrete values of $z$. Indeed, for a single dispersive Lorentz term, setting

$$
1+\hat{\chi}(z)=-1
$$

we find

$$
z= \pm \hat{\omega}, \hat{\omega}=\sqrt{\omega_{0}^{2}+\frac{\Omega^{2}}{2}}
$$

but if absorption is present, only complex solutions with negative imaginary part are obtained.

\section{TIME EVOLUTION}

In this section we extend the AFF to include magnetization. Let $\mathcal{A} \subset \mathbb{R}^{3}$ be the set containing the medium, so $\boldsymbol{\nu}_{e, m}(\mathbf{x}, \lambda)$ vanish if $\mathbf{x} \notin \mathcal{A}$ as do $\boldsymbol{F}_{2}, \boldsymbol{F}_{3}, \boldsymbol{F}_{5}$, and $\boldsymbol{F}_{6}$ below. In Ref. $6 \boldsymbol{E}$ and $\boldsymbol{B}$ were used as the electromagnetic components. It is possible to do so in the present situation, but choosing $\boldsymbol{E}$ and $\boldsymbol{H}$ gives somewhat more symmetric formulas. We introduce 


$$
\begin{gathered}
\boldsymbol{F}_{1}(\mathbf{x}, t)=\boldsymbol{E}(\mathbf{x}, t), \\
\boldsymbol{F}_{2}(\mathbf{x}, \lambda, t)=I_{\mathcal{A}}(\mathbf{x}) \int_{t_{0}}^{t} d s \sin \{\lambda(t-s)\} \boldsymbol{E}(\mathbf{x}, s), \\
\boldsymbol{F}_{3}(\mathbf{x}, \lambda, t)=I_{\mathcal{A}}(\mathbf{x}) \int_{t_{0}}^{t} d s \cos \{\lambda(t-s)\} \boldsymbol{H}(\mathbf{x}, s), \\
\boldsymbol{F}_{4}(\mathbf{x}, t)=\boldsymbol{H}(\mathbf{x}, t), \\
\boldsymbol{F}_{5}(\mathbf{x}, \lambda, t)=I_{\mathcal{A}}(\mathbf{x}) \int_{t_{0}}^{t} d s \sin \{\lambda(t-s)\} \boldsymbol{H}(\mathbf{x}, s), \\
\boldsymbol{F}_{6}(\mathbf{x}, \lambda, t)=I_{\mathcal{A}}(\mathbf{x}) \int_{t_{0}}^{t} d s \cos \{\lambda(t-s)\} \boldsymbol{E}(\mathbf{x}, s) .
\end{gathered}
$$

Then

$$
\begin{aligned}
& \boldsymbol{J}_{e}(\mathbf{x}, t)=\int d \lambda \boldsymbol{\nu}_{e}(\mathbf{x}, \lambda) \cdot \int_{t_{0}}^{t} d s \cos \{\lambda(t-s)\} \boldsymbol{F}_{1}(\mathbf{x}, s)=\int d \lambda \boldsymbol{\nu}_{e}(\mathbf{x}, \lambda) \cdot \boldsymbol{F}_{6}(\mathbf{x}, \lambda, s), \\
& \boldsymbol{J}_{m}(\mathbf{x}, t)=\int d \lambda \nu_{m}(\mathbf{x}, \lambda) \cdot \int_{t_{0}}^{t} d s \cos \{\lambda(t-s)\} \boldsymbol{F}_{4}(\mathbf{x}, s)=\int d \lambda \boldsymbol{\nu}_{m}(\mathbf{x}, \lambda) \cdot \boldsymbol{F}_{3}(\mathbf{x}, \lambda, s),
\end{aligned}
$$

and

$$
\begin{gathered}
\partial_{t} \boldsymbol{F}_{1}(\mathbf{x}, t)=\partial_{\mathbf{x}} \times \boldsymbol{F}_{4}(\mathbf{x}, t)-\int d \lambda \boldsymbol{\nu}_{e}(\mathbf{x}, \lambda) \cdot \boldsymbol{F}_{6}(\mathbf{x}, \lambda, t), \\
\partial_{t} \boldsymbol{F}_{2}(\mathbf{x}, \lambda, t)=\lambda \boldsymbol{F}_{6}(\mathbf{x}, \lambda, t), \\
\partial_{t} \boldsymbol{F}_{3}(\mathbf{x}, \lambda, t)=I_{\mathcal{A}}(\mathbf{x}) \boldsymbol{F}_{4}(\mathbf{x}, t)-\lambda \boldsymbol{F}_{5}(\mathbf{x}, \lambda, t), \\
\partial_{t} \boldsymbol{F}_{4}(\mathbf{x}, t)=-\partial_{\mathbf{x}} \times \boldsymbol{F}_{1}(\mathbf{x}, t)-\int d \lambda \boldsymbol{\nu}_{m}(\mathbf{x}, \lambda) \cdot \boldsymbol{F}_{3}(\mathbf{x}, \lambda, t), \\
\partial_{t} \boldsymbol{F}_{5}(\mathbf{x}, \lambda, t)=\lambda \boldsymbol{F}_{3}(\mathbf{x}, \lambda, t), \\
\partial_{t} \boldsymbol{F}_{6}(\mathbf{x}, \lambda, t)=I_{\mathcal{A}}(\mathbf{x}) \boldsymbol{F}_{1}(\mathbf{x}, t)-\lambda \boldsymbol{F}_{2}(\mathbf{x}, \lambda, t) .
\end{gathered}
$$

Note that

$$
\boldsymbol{F}_{2,3,5,6}\left(\mathbf{x}, \lambda, t_{0}\right)=0 .
$$

In condensed notation,

$$
\partial_{t} \boldsymbol{F}(t)=-i \mathrm{~K} \cdot \boldsymbol{F}(t) .
$$

Let $\left\langle\lambda \mid \boldsymbol{\nu}_{e, m}\right\rangle=\boldsymbol{\nu}_{e, m}(\mathbf{x}, \lambda), \boldsymbol{\epsilon}$ be the Levi-Civita symbol, and $\mathbf{p}=-i \partial_{\mathbf{x}}$ so $(\boldsymbol{\epsilon} \cdot \mathbf{p}) \cdot \mathbf{f}=i \partial_{\mathbf{x}} \times \mathbf{f}$. Then 


$$
\boldsymbol{F}=\left(\begin{array}{l}
\boldsymbol{F}_{1} \\
\boldsymbol{F}_{2} \\
\boldsymbol{F}_{3} \\
\boldsymbol{F}_{4} \\
\boldsymbol{F}_{5} \\
\boldsymbol{F}_{6}
\end{array}\right), \mathrm{K}=\left(\begin{array}{cccccc}
0 & 0 & 0 & \boldsymbol{\epsilon} \cdot \mathbf{p} & 0 & -i\left\langle\boldsymbol{\nu}_{e}\right. \\
0 & 0 & 0 & 0 & 0 & i \lambda \\
0 & 0 & 0 & i 1_{\mathcal{A}}(\mathbf{x}) & -i \lambda & 0 \\
-\boldsymbol{\epsilon} \cdot \mathbf{p} & 0 & -i\left\langle\boldsymbol{\nu}_{m}\right| & 0 & 0 & 0 \\
0 & 0 & i \lambda & 0 & 0 & 0 \\
i l_{\mathcal{A}}(\mathbf{x}) & -i \lambda & 0 & 0 & 0 & 0
\end{array}\right)=\left(\begin{array}{cc}
0 & \mathrm{~K}_{e m} \\
\mathrm{~K}_{m e} & 0
\end{array}\right) .
$$

Thus, as in the dielectric case, $\mathrm{K}$ is symplectic. Let now

$$
\begin{aligned}
& \mathcal{E}_{e}(t)=\frac{1}{2} \int d \mathbf{x} \int d \lambda \boldsymbol{\nu}_{e}(\mathbf{x}, \lambda):\left\{\boldsymbol{F}_{2}(\mathbf{x}, \lambda, t) \boldsymbol{F}_{2}(\mathbf{x}, \lambda, t)+\boldsymbol{F}_{6}(\mathbf{x}, \lambda, t) \boldsymbol{F}_{6}(\mathbf{x}, \lambda, t)\right\}, \\
& \mathcal{E}_{m}(t)=\frac{1}{2} \int d \mathbf{x} \int d \lambda \boldsymbol{\nu}_{m}(\mathbf{x}, \lambda):\left\{\boldsymbol{F}_{3}(\mathbf{x}, \lambda, t) \boldsymbol{F}_{3}(\mathbf{x}, \lambda, t)+\boldsymbol{F}_{5}(\mathbf{x}, \lambda, t) \boldsymbol{F}_{5}(\mathbf{x}, \lambda, t)\right\} .
\end{aligned}
$$

Then, with $\mathcal{E}_{e m}(t)$ as given by Eq. (2.5),

$$
\begin{gathered}
\partial_{t} \mathcal{E}_{e m}(t)=-\int d \mathbf{x}\left\{\boldsymbol{J}_{e}(\mathbf{x}, t) \cdot \boldsymbol{F}_{1}(\mathbf{x}, t)+\boldsymbol{J}_{m}(\mathbf{x}, t) \cdot \boldsymbol{F}_{4}(\mathbf{x}, t)\right\}, \\
\partial_{t} \mathcal{E}_{e}(t)=\int d \mathbf{x} \int d \lambda \boldsymbol{\nu}_{e}(\mathbf{x}, \lambda): \boldsymbol{F}_{6}(\mathbf{x}, \lambda, t) \boldsymbol{F}_{1}(\mathbf{x}, t)=\int d \mathbf{x} \boldsymbol{J}_{e}(\mathbf{x}, t) \cdot \boldsymbol{F}_{1}(\mathbf{x}, t), \\
\partial_{t} \mathcal{E}_{m}(t)=\int d \mathbf{x} \int d \lambda \boldsymbol{\nu}_{m}(\mathbf{x}, \lambda): \boldsymbol{F}_{5}(\mathbf{x}, \lambda, t) \boldsymbol{F}_{4}(\mathbf{x}, t)=\int d \mathbf{x} \boldsymbol{J}_{m}(\mathbf{x}, t) \cdot \boldsymbol{F}_{4}(\mathbf{x}, t),
\end{gathered}
$$

so, with

$$
\mathcal{E}(t)=\mathcal{E}_{\text {em }}(t)+\mathcal{E}_{e}(t)+\mathcal{E}_{m}(t),
$$

we have

$$
\partial_{t} \mathcal{E}(t)=0,
$$

i.e., $\mathcal{E}(t)$ is conserved in time. At this point we note that the standard expression for the conserved energy is

$$
\begin{aligned}
\partial_{t} \mathcal{E}= & \int d \mathbf{x}\left\{\boldsymbol{E}(\mathbf{x}, t) \cdot \partial_{t} \boldsymbol{D}(\mathbf{x}, t)+\boldsymbol{H}(\mathbf{x}, t) \cdot \partial_{t} \boldsymbol{B}(\mathbf{x}, t)\right\}=\partial_{t} \mathcal{E}_{e m}(t)+\int d \mathbf{x}\left\{\boldsymbol{E}(\mathbf{x}, t) \cdot \boldsymbol{J}_{e}(\mathbf{x}, t)\right. \\
& \left.+\boldsymbol{H}(\mathbf{x}, t) \cdot \boldsymbol{J}_{m}(\mathbf{x}, t)\right\},
\end{aligned}
$$

so the two expressions agree.

We introduce the inner product, 


$$
\begin{aligned}
(\boldsymbol{F}, \boldsymbol{G})= & \int d \mathbf{x}\left[\boldsymbol{F}_{1}(\mathbf{x}) \cdot \overline{\boldsymbol{G}_{1}(\mathbf{x})}+\boldsymbol{F}_{4}(\mathbf{x}) \cdot \overline{\boldsymbol{G}_{4}(\mathbf{x})}\right]+\int_{\mathcal{A}} d \mathbf{x} \int d \lambda \boldsymbol{\nu}_{e}(\mathbf{x}, \lambda):\left[\boldsymbol{F}_{2}(\mathbf{x}, \lambda) \overline{\boldsymbol{G}_{2}(\mathbf{x}, \lambda)}\right. \\
& \left.+\boldsymbol{F}_{6}(\mathbf{x}, \lambda) \overline{\boldsymbol{G}_{6}(\mathbf{x}, \lambda)}\right]+\int_{\mathcal{A}} d \mathbf{x} \int d \lambda \boldsymbol{\nu}_{m}(\mathbf{x}, \lambda):\left[\boldsymbol{F}_{3}(\mathbf{x}, \lambda) \overline{\boldsymbol{G}_{3}(\mathbf{x}, \lambda)}+\boldsymbol{F}_{5}(\mathbf{x}, \lambda) \overline{\boldsymbol{G}_{5}(\mathbf{x}, \lambda)}\right]
\end{aligned}
$$

which defines the Hilbert space

$$
\begin{gathered}
\mathcal{K}=\oplus_{j=1}^{6} \mathcal{K}_{j}, \mathcal{K}_{1}=\mathcal{K}_{4}=L^{2}\left(\mathbb{R}^{3}, d \mathbf{x} ; \mathbb{C}^{3}\right) \\
\mathcal{K}_{2}=\mathcal{K}_{6}=L^{2}\left(\mathcal{A}, d \mathbf{x} ; \mathbb{C}^{3}\right) \otimes L^{2}\left(\mathbb{R}, \boldsymbol{\nu}_{e} d \lambda\right), \mathcal{K}_{3}=\mathcal{K}_{5}=L^{2}\left(\mathcal{A}, d \mathbf{x} ; \mathbb{C}^{3}\right) \otimes L^{2}\left(\mathbb{R}, \boldsymbol{\nu}_{m} d \lambda\right)
\end{gathered}
$$

Then

$$
\|\boldsymbol{F}(t)\|^{2}=(\boldsymbol{F}(t), \boldsymbol{F}(t))=2 \mathcal{E}
$$

is conserved in time. In Appendices $\mathrm{A}$ and $\mathrm{C}$ it is shown (for simpler notation the isotropic case is considered) that $\mathrm{K}$ is self-adjoint in $\mathcal{K}$ under some mild conditions on the susceptibilities $\left(\left|\boldsymbol{\chi}_{e, m}^{\prime}(\mathbf{x}, 0)\right| \leq c<\infty\right)$, which we assume to hold from now on. In fact, in

$$
\mathrm{K}=\mathrm{K}_{0}+\mathrm{K}_{1} \text {, }
$$

where

$$
\mathrm{K}_{0}=\left(\begin{array}{cccccc}
0 & 0 & 0 & \boldsymbol{\epsilon} \cdot \mathbf{p} & 0 & 0 \\
0 & 0 & 0 & 0 & 0 & i \lambda \\
0 & 0 & 0 & 0 & -i \lambda & 0 \\
-\boldsymbol{\epsilon} \cdot \mathbf{p} & 0 & 0 & 0 & 0 & 0 \\
0 & 0 & i \lambda & 0 & 0 & 0 \\
0 & -i \lambda & 0 & 0 & 0 & 0
\end{array}\right), \mathrm{K}_{1}=\left(\begin{array}{cccccc}
0 & 0 & 0 & 0 & 0 & -i\left\langle\boldsymbol{\nu}_{e}\right| \\
0 & 0 & 0 & 0 & 0 & 0 \\
0 & 0 & 0 & i & 0 & 0 \\
0 & 0 & -i\left\langle\boldsymbol{\nu}_{m}\right| & 0 & 0 & 0 \\
0 & 0 & 0 & 0 & 0 & 0 \\
i & 0 & 0 & 0 & 0 & 0
\end{array}\right)
$$

$\mathrm{K}_{1}$ is a bounded self-adjoint operator. Thus we are dealing with a unitary time evolution on $\mathcal{K}$,

$$
\mathrm{W}(t)=\exp [-i \mathrm{~K} t]
$$

As mentioned before it implies that the time evolution of the electromagnetic fields is properly defined. In case the electromagnetic fields are square integrable at the initial time, this remains true at all later times (note the passivity condition above). This is not evident in the original formulation which contains time convolutions.

Remark: Note that passivity is not required to obtain the conservation of $\mathcal{E}(t)$. Since $\mathcal{E}_{\text {em }}(0)$ $\geq 0$ and $\mathcal{E}_{e}(0)=\mathcal{E}_{m}(0)=0$ we still have $\mathcal{E}(t)=\mathcal{E}(0) \geq 0$. However, without it, $\mathcal{E}_{e m}(t)$ may increase in time and $\mathcal{E}_{e}(t)$ and $\mathcal{E}_{m}(t)$ become negative for $t>0\left[\boldsymbol{\nu}_{e, m}(\mathbf{x}, \lambda)\right.$ may no longer be non-negative $]$. We still can introduce the inner product $(\boldsymbol{F}, \boldsymbol{G})$ but the associated norm is also no longer non-negative definite.

For dispersive systems $\boldsymbol{\nu}_{e, m}(\mathbf{x}, \lambda)$ becomes a sum of $\delta$-functions. Let us assume that only one dispersive Lorentz contribution is present in both $\chi$ 's and that the medium is homogeneous and isotropic over $\mathcal{A}$. Then $\nu_{e, m}(\mathbf{x})=\nu_{e, m} \mathrm{I}_{\mathcal{A}}(\mathbf{x})$, so in $\mathcal{K}_{2,3,5,6}$ the $\mathbf{x}$-integration is over $\mathcal{A}$ and 


$$
\begin{gathered}
\chi_{e}(\mathbf{x}, t)=\Omega_{e}^{2} I_{\mathcal{A}}(\mathbf{x}) \frac{\sin \lambda_{e} t}{\lambda_{e}}, \quad \chi_{e}^{\prime}(\mathbf{x}, t)=\Omega_{e}^{2} \mathrm{I}_{\mathcal{A}}(\mathbf{x}) \cos \lambda_{e} t, \\
\chi_{m}(\mathbf{x}, t)=\Omega_{m}^{2} \mathrm{I}_{\mathcal{A}}(\mathbf{x}) \frac{\sin \lambda_{m} t}{\lambda_{m}}, \quad \chi_{m}^{\prime}(\mathbf{x}, t)=\Omega_{m}^{2} \mathrm{I}_{\mathcal{A}}(\mathbf{x}) \cos \lambda_{m} t, \\
\hat{\chi}_{e}(\mathbf{x}, z)=\frac{\Omega_{e}^{2}}{\lambda_{e}^{2}-z^{2}} \mathrm{I}_{\mathcal{A}}(\mathbf{x}), \quad \hat{\chi}_{m}(\mathbf{x}, z)=\frac{\Omega_{m}^{2}}{\lambda_{m}^{2}-z^{2}} \mathrm{I}_{\mathcal{A}}(\mathbf{x}),
\end{gathered}
$$

so

$$
\begin{gathered}
\boldsymbol{J}_{e}(\mathbf{x}, t)=\Omega_{e}^{2} \mathrm{I}_{\mathcal{A}}(\mathbf{x}) \int_{t_{0}}^{t} d s \cos \left\{\lambda_{e}(t-s)\right\} \boldsymbol{E}(\mathbf{x}, s), \\
\boldsymbol{J}_{m}(\mathbf{x}, t)=\Omega_{m}^{2} I_{\mathcal{A}}(\mathbf{x}) \int_{t_{0}}^{t} d s \cos \left\{\lambda_{m}(t-s)\right\} \boldsymbol{H}(\mathbf{x}, s) .
\end{gathered}
$$

In this case, with $\boldsymbol{F}_{1}=\boldsymbol{E}, \boldsymbol{F}_{4}=\boldsymbol{H}$, and

$$
\begin{aligned}
& \boldsymbol{F}_{2}(\mathbf{x}, t)=I_{\mathcal{A}}(\mathbf{x}) \int_{t_{0}}^{t} d s \sin \left\{\lambda_{e}(t-s)\right\} \boldsymbol{E}(\mathbf{x}, s), \quad \boldsymbol{F}_{3}(\mathbf{x}, t)=\mathrm{I}_{\mathcal{A}}(\mathbf{x}) \int_{t_{0}}^{t} d s \cos \left\{\lambda_{m}(t-s)\right\} \boldsymbol{H}(\mathbf{x}, s), \\
& \boldsymbol{F}_{5}(\mathbf{x}, t)=\mathrm{I}_{\mathcal{A}}(\mathbf{x}) \int_{t_{0}}^{t} d s \sin \left\{\lambda_{m}(t-s)\right\} \boldsymbol{H}(\mathbf{x}, s), \quad \boldsymbol{F}_{6}(\mathbf{x}, t)=\mathrm{I}_{\mathcal{A}}(\mathbf{x}) \int_{t_{0}}^{t} d s \cos \left\{\lambda_{e}(t-s)\right\} \boldsymbol{E}(\mathbf{x}, s),
\end{aligned}
$$

once more,

$$
\partial_{t} \boldsymbol{F}(t)=-i \mathbf{K} \cdot \boldsymbol{F}(t)
$$

where now

$$
\mathrm{K}=\left(\begin{array}{cccccc}
0 & 0 & 0 & \boldsymbol{\epsilon} \cdot \mathbf{p} & 0 & -i \Omega_{e}^{2} \\
0 & 0 & 0 & 0 & 0 & i \lambda_{e} \\
0 & 0 & 0 & i 1_{\mathcal{A}}(\mathbf{x}) & -i \lambda_{m} & 0 \\
-\boldsymbol{\epsilon} \cdot \mathbf{p} & 0 & -i \Omega_{m}^{2} & 0 & 0 & 0 \\
0 & 0 & i \lambda_{m} & 0 & 0 & 0 \\
i 1_{\mathcal{A}}(\mathbf{x}) & -i \lambda_{e} & 0 & 0 & 0 & 0
\end{array}\right)
$$

whereas $\mathcal{K}_{2,6}$ and $\mathcal{K}_{3,5}$ reduce to $L^{2}\left(\mathcal{A}, \Omega_{e}^{2} d \mathbf{x} ; \mathrm{C}^{3}\right)$ and $L^{2}\left(\mathcal{A}, \Omega_{m}^{2} d \mathbf{x} ; \mathrm{C}^{3}\right)$, respectively. In case there are more dispersive Lorentz terms in the susceptibilities the number of auxiliary fields increases accordingly.

\section{LAPLACE-TRANSFORMED FIELDS}

The equations of motion can equivalently be expressed in terms of Laplace transforms. Setting $t_{0}=0$, we obtain 


$$
\hat{\boldsymbol{F}}(z)=i[z-\mathrm{K}]^{-1} \cdot \boldsymbol{F}(0)=i \mathrm{R}(z) \cdot \boldsymbol{F}(0), \operatorname{Im} z>0 .
$$

From this the relations for the various components of $\hat{\boldsymbol{F}}(z)$ can be obtained in terms of those of $\boldsymbol{F}(0)$ by projecting upon the appropriate subspace, see Appendices A and C. However, a direct approach involves less calculations. Thus,

$$
\begin{aligned}
& -i z \hat{\boldsymbol{D}}(\mathbf{x}, z)-\boldsymbol{D}(\mathbf{x}, 0)=-i(\boldsymbol{\epsilon} \cdot \mathbf{p}) \cdot \hat{\boldsymbol{H}}(\mathbf{x}, z), \\
& -i z \hat{\boldsymbol{B}}(\mathbf{x}, z)-\boldsymbol{B}(\mathbf{x}, 0)=+i(\boldsymbol{\epsilon} \cdot \mathbf{p}) \cdot \hat{\boldsymbol{E}}(\mathbf{x}, z) .
\end{aligned}
$$

Since

$$
\hat{\boldsymbol{D}}(\mathbf{x}, z)=\boldsymbol{\varepsilon}(\mathbf{x}, z) \cdot \hat{\boldsymbol{E}}(\mathbf{x}, z), \hat{\boldsymbol{B}}(\mathbf{x}, z)=\boldsymbol{\mu}(\mathbf{x}, z) \cdot \hat{\boldsymbol{H}}(\mathbf{x}, z),
$$

we obtain, noting that $\boldsymbol{D}(\mathbf{x}, 0)=\boldsymbol{E}(\mathbf{x}, 0)$ and $\boldsymbol{H}(\mathbf{x}, 0)=\boldsymbol{B}(\mathbf{x}, 0)$,

$$
\mathrm{L}^{e}(z) \cdot \hat{\boldsymbol{E}}(\mathbf{x}, z)=\mathbf{g}^{e}(\mathbf{x}, z), \quad \mathrm{L}^{m}(z) \cdot \hat{\boldsymbol{H}}(\mathbf{x}, z)=\mathbf{g}^{m}(\mathbf{x}, z),
$$

where

$$
\begin{gathered}
\mathrm{L}^{e}(z)=z^{2} \boldsymbol{\varepsilon}(\mathbf{x}, z)+(\boldsymbol{\epsilon} \cdot \mathbf{p}) \cdot \boldsymbol{\mu}(\mathbf{x}, z)^{-1} \cdot(\boldsymbol{\epsilon} \cdot \mathbf{p}), \\
\mathrm{L}^{m}(z)=z^{2} \boldsymbol{\mu}(\mathbf{x}, z)+(\boldsymbol{\epsilon} \cdot \mathbf{p}) \cdot \boldsymbol{\varepsilon}(\mathbf{x}, z)^{-1} \cdot(\boldsymbol{\epsilon} \cdot \mathbf{p}), \\
\mathbf{g}^{e}(\mathbf{x}, z)=i z \boldsymbol{E}(\mathbf{x}, 0)+i(\boldsymbol{\epsilon} \cdot \mathbf{p}) \cdot\left\{\boldsymbol{\mu}(\mathbf{x}, z)^{-1} \cdot \boldsymbol{H}(\mathbf{x}, 0)\right\}, \\
\mathbf{g}^{m}(\mathbf{x}, z)=i z \boldsymbol{H}(\mathbf{x}, 0)-i(\boldsymbol{\epsilon} \cdot \mathbf{p}) \cdot\left\{\boldsymbol{\varepsilon}(\mathbf{x}, z)^{-1} \cdot \boldsymbol{E}(\mathbf{x}, 0)\right\} .
\end{gathered}
$$

Here $L^{e}(z)$ and $L^{m}(z)$ are the electric and magnetic Helmholtz operators. Let now

$$
\mathbf{R}^{e}(z)=\mathrm{L}^{e}(z)^{-1}, \mathbf{R}^{m}(z)=\mathrm{L}^{m}(z)^{-1} .
$$

Then

$$
\hat{\boldsymbol{E}}(\mathbf{x}, z)=\mathrm{R}^{e}(z) \cdot \mathbf{g}^{e}(\mathbf{x}, z), \quad \hat{\boldsymbol{H}}(\mathbf{x}, z)=\mathrm{R}^{m}(z) \cdot \mathbf{g}^{m}(\mathbf{x}, z) .
$$

Note that

$$
\partial_{\mathbf{x}} \cdot L^{e}(z) \cdot \hat{\boldsymbol{E}}(\mathbf{x}, z)=z^{2} \partial_{\mathbf{x}} \cdot\{\boldsymbol{\varepsilon}(\mathbf{x}, z) \cdot \hat{\boldsymbol{E}}(\mathbf{x}, z)\}=z^{2} \partial_{\mathbf{x}} \cdot \hat{\boldsymbol{D}}(\mathbf{x}, z)=i z \partial_{\mathbf{x}} \cdot \boldsymbol{E}(\mathbf{x}, 0)=0,
$$

as it should be.

We can make the identification (see Appendices A and C),

$$
\mathrm{P}_{1} \mathrm{R}(z) \mathrm{P}_{1}=z \mathrm{R}^{e}(z) \mathrm{P}_{1} .
$$

Since the left hand side is a bounded operator, it follows that $\operatorname{R}^{e}(z), \operatorname{Im} z>0$, has a closed densely defined extension, which is, in fact, bounded and the same is true for $\mathrm{R}^{m}(z)$. Note that, since $\overline{\boldsymbol{\varepsilon}(\mathbf{x}, z)}=\boldsymbol{\varepsilon}(\mathbf{x},-\bar{z})$ and $\overline{\boldsymbol{\mu}(\mathbf{x}, z)}=\boldsymbol{\mu}(\mathbf{x},-\bar{z}), \mathbf{R}^{e}(z)^{*}=\mathbf{R}^{e}(-\bar{z})$. Next we introduce Green's functions,

$$
\mathbf{G}^{e, m}(\mathbf{x}, \mathbf{y}, z)=\left\langle\mathbf{x}\left|\mathbf{R}^{e, m}(z)\right| \mathbf{y}\right\rangle .
$$

They are square integrable in $\mathbf{x}$ and $\mathbf{y}$, respectively, are analytic in the open upper half plane and have the following further properties: 


$$
\mathbf{G}(\mathbf{x}, \mathbf{y}, z)=\mathbf{G}(\mathbf{y}, \mathbf{x},-\bar{z})^{\dagger}, \frac{1}{2 \pi} \int_{\Gamma} d z \mathbf{G}(\mathbf{x}, \mathbf{y}, z)=0, \frac{1}{2 \pi i} \int_{\Gamma} d z z \mathbf{G}(\mathbf{x}, \mathbf{y}, z)=-\delta(\mathbf{x}-\mathbf{y}) \mathrm{U} .
$$

Now

$$
\mathrm{L}^{e, m} \cdot \mathrm{G}^{e, m}(\mathbf{x}, \mathbf{y}, z)=\delta(\mathbf{x}-\mathbf{y}) \mathrm{U} .
$$

Note that, although $\partial_{\mathbf{x}} \cdot L^{e} \cdot \hat{\boldsymbol{E}}(\mathbf{x}, z)=0$, this is a special case since

$$
\partial_{\mathbf{x}} \cdot \mathrm{L}_{\mathbf{x}}^{e} \cdot \int d \mathbf{y G}(\mathbf{x}, \mathbf{y}, z) \cdot \mathbf{h}(y)=\partial_{\mathbf{x}} \cdot \mathbf{h}(\mathbf{x}),
$$

which need not vanish for general $\mathbf{h}$.

The spatially piecewise constant situation is the case where $\mathbb{R}^{3}=\left\{\cup_{j} \mathcal{M}_{j}\right\} \cup\{$ interfaces $\}$, with the $\mathcal{M}_{j}$ 's disjoint open sets separated by sufficiently regular interfaces (so boundary conditions can be imposed), whereas the susceptibilities are constant over $\mathcal{M}_{j}$,

$$
\boldsymbol{\varepsilon}(\mathbf{x}, z)=\boldsymbol{\varepsilon}_{j}(z), \boldsymbol{\mu}(\mathbf{x}, z)=\boldsymbol{\mu}_{j}(z), \mathbf{x} \in \mathcal{M}_{j} .
$$

\section{LAYERED SYSTEMS}

\section{A. General}

In the sequel we only consider the electric Green's function and we drop the superscript $e$. We also assume that the system is isotropic (in the anisotropic case the reduction to an expression featuring the scalar Green's functions for $s$ and $p$ polarization is not possible, in general). Here we consider the situation that the $\mathcal{M}_{j}$ 's are a number of layers parallel to the $X_{1} X_{2}$-plane. Then the permeabilities only depend on $x_{3}$,

$$
\varepsilon(\mathbf{x}, z)=\varepsilon\left(x_{3}, z\right), \mu(\mathbf{x}, z)=\mu\left(x_{3}, z\right) .
$$

We exploit the translational invariance in the $X_{1}$ and $X_{2}$ directions. Let $\mathbf{k}=\left(k_{1}, k_{2}, k_{3}\right)$ and $\boldsymbol{\kappa}$ $=\kappa \mathbf{e}_{\boldsymbol{\kappa}}=\mathbf{k}^{\perp}=\left(k_{1}, k_{2}, 0\right) \perp \mathbf{e}_{3}$,

$$
\begin{gathered}
\mathbf{G}(\mathbf{x}, \mathbf{y}, z)=(2 \pi)^{-2} \int d \boldsymbol{\kappa} \exp \left[-i \boldsymbol{\kappa} \cdot\left(\mathbf{x}^{\perp}-\mathbf{y}^{\perp}\right)\right] \mathbf{G}_{\boldsymbol{\kappa}}\left(x_{3}, y_{3}, z\right), \\
\mathbf{G}_{\boldsymbol{\kappa}}\left(x_{3}, y_{3}, z\right)=(2 \pi)^{-2} \int d \mathbf{x}^{\perp} \exp \left[i \boldsymbol{\kappa} \cdot\left(\mathbf{x}^{\perp}-\mathbf{y}^{\perp}\right)\right] \mathbf{G}(\mathbf{x}, \mathbf{y}, z), \\
\mathbf{m}_{\boldsymbol{\kappa}}\left(x_{3}, z\right)=\int d \mathbf{x}^{\perp} \exp \left[i \boldsymbol{\kappa} \cdot \mathbf{x}^{\perp}\right] \mathbf{m}(\mathbf{x}, z),
\end{gathered}
$$

where $\mathbf{m}$ can be $\boldsymbol{E}, \boldsymbol{B}, \mathbf{g}^{e, m}$, etc. Then

$$
\begin{aligned}
\boldsymbol{E}(\mathbf{x}, t) & =(2 \pi)^{-1} \int_{\Gamma} d z \exp [-i z t] \hat{\boldsymbol{E}}(\mathbf{x}, z) \\
& =(2 \pi)^{-3} \int_{\Gamma} d z \exp [-i z t] \int d \boldsymbol{\kappa} \exp \left[-i \boldsymbol{\kappa} \cdot\left(\mathbf{x}^{\perp}-\mathbf{y}^{\perp}\right)\right] \int d y_{3} \mathbf{G}_{\boldsymbol{\kappa}}\left(x_{3}, y_{3}, z\right) \cdot \mathbf{g}_{\boldsymbol{\kappa}}\left(y_{3}, z\right) .
\end{aligned}
$$

Omitting the subscript 3 in $x_{3}$, etc., from now on, $\hat{\boldsymbol{E}}_{\boldsymbol{\kappa}}(x, z) \in \mathcal{H}=L^{2}\left(\mathbb{R}, d x ; \mathrm{C}^{3}\right)$, 


$$
\begin{gathered}
\mathbf{g}_{\boldsymbol{\kappa}}(y, z)=i z \boldsymbol{E}_{\boldsymbol{\kappa}}(y, 0)-\left(i \boldsymbol{\kappa}+\partial_{y} \mathbf{e}_{3}\right) \times \frac{1}{\mu(y, z)} \boldsymbol{B}_{\boldsymbol{\kappa}}(y, 0), \\
\mathrm{L}_{\boldsymbol{\kappa}} \cdot \hat{\boldsymbol{E}}_{\boldsymbol{\kappa}}(x, z)=\mathbf{g}_{\boldsymbol{\kappa}}(x, z),
\end{gathered}
$$

and

$$
\mathbf{G}_{\boldsymbol{\kappa}}(x, y, z)=\left\langle x\left|\mathbf{L}_{\boldsymbol{\kappa}}^{-1}\right| y\right\rangle, \mathbf{L}_{\boldsymbol{\kappa}} \cdot \mathbf{G}_{\boldsymbol{\kappa}}(x, y, z)=\delta(x-y) \mathrm{U} .
$$

$\mathrm{L}_{\boldsymbol{\kappa}}$ is obtained from $\mathrm{L}^{e}$ by replacing $\mathbf{p}$ by $\boldsymbol{\kappa}+p \mathbf{e}_{3}=\kappa \mathbf{e}_{\boldsymbol{\kappa}}+p \mathbf{e}_{3}, p=-i \partial_{x}$. Denoting

$$
\zeta(x, \kappa, z)^{2}=z^{2} \varepsilon(x, z) \mu(x, z)-\kappa^{2},
$$

we obtain

$$
\begin{aligned}
\mathrm{L}_{\boldsymbol{\kappa}}= & \left\{\frac{\zeta(x, \kappa, z)^{2}}{\mu(\mathbf{x}, z)}-p \frac{1}{\mu(\mathbf{x}, z)} p\right\} \mathbf{e}_{3} \times \mathbf{e}_{\boldsymbol{\kappa}} \mathbf{e}_{3} \times \mathbf{e}_{\boldsymbol{\kappa}}+\left\{z^{2} \varepsilon(\mathbf{x}, z)-p \frac{1}{\mu(\mathbf{x}, z)} p\right\} \mathbf{e}_{\boldsymbol{\kappa}} \mathbf{e}_{\boldsymbol{\kappa}}+\frac{\zeta(x, \kappa, z)^{2}}{\mu(\mathbf{x}, z)} \mathbf{e}_{3} \mathbf{e}_{3} \\
& +p \frac{\kappa}{\mu(\mathbf{x}, z)} \mathbf{e}_{\boldsymbol{\kappa}} \mathbf{e}_{3}+\frac{\kappa}{\mu(\mathbf{x}, z)} p \mathbf{e}_{3} \mathbf{e}_{\boldsymbol{\kappa}}=\mathrm{L}_{\boldsymbol{\kappa}}^{s}+\mathrm{L}_{\boldsymbol{\kappa}}^{p}
\end{aligned}
$$

where $L_{\boldsymbol{\kappa}}^{s}$ is the $s$-polarization part (the term with $\mathbf{e}_{3} \times \mathbf{e}_{\boldsymbol{\kappa}} \mathbf{e}_{3} \times \mathbf{e}_{\boldsymbol{\kappa}}$ ) and $L_{\boldsymbol{\kappa}}^{p}$, the $p$-polarization part, the remainder. The corresponding decomposition for $\mathrm{G}_{\boldsymbol{\kappa}}$ is

$$
\mathrm{G}_{\boldsymbol{\kappa}}(x, y, z)=\mathrm{G}_{\boldsymbol{\kappa}}^{s}(x, y, z)+\mathrm{G}_{\boldsymbol{\kappa}}^{p}(x, y, z)=\left\langle x\left|\left(\mathrm{~L}_{\boldsymbol{\kappa}}^{s}\right)^{-1}\right| y\right\rangle+\left\langle x\left|\left(\mathrm{~L}_{\boldsymbol{\kappa}}^{p}\right)^{-1}\right| y\right\rangle .
$$

It is customary to consider the scalar Green's functions associated with the electric and magnetic $s$-polarization parts. However, the latter is transverse, whereas $\mathrm{G}_{\boldsymbol{\kappa}}^{p}$ also contains a longitudinal component. In addition, in obtaining atomic radiative decay rates, the full tensorial expression for Green's function is required and there is no simple relation between (the transverse part of) $\mathrm{G}_{\boldsymbol{\kappa}}^{p}$ and the magnetic s-polarized Green's function. Thus we calculated $\mathrm{G}_{\boldsymbol{\kappa}}^{p}$ in Appendices A and $\mathrm{C}$ with the result

$$
\begin{gathered}
\mathbf{G}_{\boldsymbol{\kappa}}(x, y, z)=\mathrm{G}_{s}(x, y, z, \kappa)+\mathrm{G}_{p}(x, y, z, \kappa), \\
\mathbf{G}_{s}(x, y, z, \kappa)=G_{s}(x, y, z, \kappa) \mathbf{e}_{3} \times \mathbf{e}_{\boldsymbol{\kappa}} \mathbf{e}_{3} \times \mathbf{e}_{\boldsymbol{\kappa}}, \\
\mathbf{G}_{p}(x, y, z, \kappa)=\left(\mathbf{e}_{\boldsymbol{\kappa}}+\frac{i \kappa}{\zeta(x)^{2}} \partial_{x} \mathbf{e}_{3}\right)\left(\mathbf{e}_{\boldsymbol{\kappa}}-\frac{i \kappa}{\zeta(y)^{2}} \partial_{y} \mathbf{e}_{3}\right) G_{p}(x, y, z, \kappa),
\end{gathered}
$$

where $G_{s}$ and $G_{p}$ satisfy

$$
\begin{aligned}
& \left\{z^{2} \varepsilon(x, z)-p \frac{z^{2} \varepsilon(x, z)}{\zeta(x, \kappa, z)^{2}} p\right\} G_{p}(x, y, z, \kappa)=\delta(x-y), \\
& \left\{\frac{\zeta(x, \kappa, z)^{2}}{\mu(x, z)}-p \frac{1}{\mu(x, z)} p\right\} G_{s}(x, y, z, \kappa)=\delta(x-y) .
\end{aligned}
$$

In order to obtain $G_{p}$ and $G_{s}$ we have to supplement these differential equations with the boundary conditions at an interface. Since $\partial_{x}\left(\varepsilon / \zeta^{2}\right) \partial_{x} G_{p}$ must make sense, $\partial_{x} G_{p}$ must exist, so we can choose $G_{p}$ to be continuous in $x$. In addition $\left(\varepsilon / \zeta^{2}\right) \partial_{x} G_{p}$ must be differentiable, so it must also be continuous in $x$. Similarly we find that $G_{s}$ must be continuous in $x$, as well as $\mu^{-1} \partial_{x} G_{s}$ and again the same applies with $x$ and $y$ interchanged. These boundary conditions can be shown to correspond to the usual boundary conditions for $\boldsymbol{D}$ and $\boldsymbol{E}$. In addition, in view of the square integra- 
bility in $x$ and $y$, there are no exponentially increasing contributions for layers that extend to $x$ $= \pm \infty$.

\section{B. Two half spaces filled with different materials}

We consider the situation where the half spaces $x>0$ and $x<0$ are filled with media characterized according to

$$
\varepsilon(x, z)=\left\{\begin{array}{ll}
\varepsilon_{+}(z), & x>0 \\
\varepsilon_{-}(z), & x<0,
\end{array} \quad \mu(x, z)= \begin{cases}\mu_{+}(z), & x>0 \\
\mu_{-}(z), & x<0\end{cases}\right.
$$

We denote

$$
\begin{gathered}
\zeta_{+}(\kappa, z)^{2}=z^{2} \varepsilon_{+}(z) \mu_{+}(z)-\kappa^{2}, \quad \zeta_{-}(\kappa, z)^{2}=z^{2} \varepsilon_{-}(z) \mu_{-}(z)-\kappa^{2}, \\
K_{ \pm}(\kappa, z)=\frac{\zeta_{ \pm}(\kappa, z)}{2 i z^{2} \varepsilon_{ \pm}(z)}, \quad L_{ \pm}(\kappa, z)=\frac{\mu_{ \pm}(z)}{2 i \zeta_{ \pm}(\kappa, z)}
\end{gathered}
$$

and introduce the Fresnel reflection coefficients,

$$
r_{p}=\frac{\varepsilon_{-} \zeta_{+}-\varepsilon_{+} \zeta_{-}}{\varepsilon_{-} \zeta_{+}+\varepsilon_{+} \zeta_{-}}, \quad r_{s}=\frac{\mu_{-} \zeta_{+}-\mu_{+} \zeta_{-}}{\mu_{-} \zeta_{+}+\mu_{+} \zeta_{-}} .
$$

Using square integrability in $x$ and $y$ and the boundary conditions on the interface, we obtain in the usual way

$$
\begin{aligned}
G_{p}(x, y, z)= & K_{+}\left\{\exp \left[i \zeta_{+}|x-y|\right]-r_{p} \exp \left[i \zeta_{+}(x+y)\right]\right\} \theta(x) \theta(y)+\frac{1}{i z^{2}} \frac{\zeta_{+} \zeta_{-}}{\varepsilon_{+} \zeta_{-}+\varepsilon_{-} \zeta_{+}} \\
& \times\left\{\exp \left[i \zeta_{+} x-i \zeta_{-} y\right] \theta(x) \theta(-y)+\exp \left[-i \zeta_{-} x+i \zeta_{+} y\right] \theta(-x) \theta(y)\right\} \\
& +K_{-}\left\{\exp \left[i \zeta_{-}|x-y|\right]+r_{p} \exp \left[-i \zeta_{-}(x+y)\right]\right\} \theta(-x) \theta(-y)
\end{aligned}
$$

and

$$
\begin{aligned}
G_{s}(x, y, z)= & L_{+}\left\{\exp \left[i \zeta_{+}|x-y|\right]+r_{s} \exp \left[i \zeta_{+}(x+y)\right]\right\} \theta(x) \theta(y)-i \frac{\mu_{+} \mu_{-}}{\mu_{+} \zeta_{-}+\mu_{-} \zeta_{+}} \\
& \times\left\{\exp \left[i \zeta_{+} x-i \zeta_{-} y\right] \theta(x) \theta(-y)+\exp \left[-i \zeta_{-} x+i \zeta_{+} y\right] \theta(-x) \theta(y)\right\} \\
& +L_{-}\left\{\exp \left[i \zeta_{-}|x-y|\right]-r_{s} \exp \left[-i \zeta_{-}(x+y)\right]\right\} \theta(-x) \theta(-y),
\end{aligned}
$$

from which the $x$ and $y$ derivatives of $G_{p}(x, y, z)$, etc., present in $\mathrm{G}_{p}(x, y, z, \kappa)$, can be obtained in explicit form.

The case where the region $x>0$ consists of vacuum and the initial state is contained in this region is of particular interest. It applies to the situation where an electromagnetic wavepacket in vacuum is traveling toward the medium. Then, labeling vacuum quantities with the subscript 0 and deleting the subscript—-for quantities associated with the medium,

$$
\begin{gathered}
K_{+}(\kappa, z)=K_{0}(\kappa, z)=\frac{\zeta_{0}(\kappa, z)}{2 i z^{2}}, \quad L_{+}(\kappa, z)=L_{0}(\kappa, z)=\frac{1}{2 i \zeta_{0}(\kappa, z)}, \\
\zeta_{0}(\kappa, z)=\sqrt{z^{2}-\kappa^{2}}, \quad r_{p 0}=\frac{\varepsilon \zeta_{0}-\zeta}{\varepsilon \zeta_{0}+\zeta}, \quad r_{s 0}=\frac{\mu \zeta_{0}-\zeta}{\mu \zeta_{0}+\zeta},
\end{gathered}
$$

so, for $y>0$, 


$$
\begin{gathered}
G_{p}(x, y, z)=K_{0}\left\{\exp \left[i \zeta_{0}|x-y|\right]-r_{p 0} \exp \left[i \zeta_{0}(x+y)\right]\right\} \theta(x)+\frac{1}{i z^{2}} \frac{\zeta_{0} \zeta}{\zeta+\varepsilon \zeta_{0}} \exp \left[-i \zeta x+i \zeta_{0} y\right] \theta(-x), \\
G_{s}(x, y, z)=L_{0}\left\{\exp \left[i \zeta_{0}|x-y|\right]+r_{s 0} \exp \left[i \zeta_{0}(x+y)\right]\right\} \theta(x)-i \frac{\mu}{\zeta+\mu \zeta_{0}} \exp \left[-i \zeta x+i \zeta_{0} y\right] \theta(-x) .
\end{gathered}
$$

\section{THE NIM SITUATION}

We continue our investigation of the half space case, assuming that

$$
\varepsilon(z)=\mu(z)=1-\frac{\Omega^{2}}{z^{2}-\omega_{0}^{2}},
$$

the dispersive Lorentz case, and rewrite

$$
\Phi(z)=\frac{1}{\zeta(\kappa, z)+\varepsilon(z) \zeta_{0}(\kappa, z)}=\frac{\zeta-\varepsilon \zeta_{0}}{\zeta^{2}-\varepsilon^{2} \zeta_{0}^{2}}=\frac{\zeta-\varepsilon \zeta_{0}}{\left(z^{2} \varepsilon^{2}-\kappa^{2}\right)-\varepsilon^{2}\left(z^{2}-\kappa^{2}\right)}=\frac{\zeta-\varepsilon \zeta_{0}}{\kappa^{2}} \frac{1}{\varepsilon-1} \frac{1}{\varepsilon+1}
$$

Note that this expression can become infinite if $\varepsilon^{2}=1$. In the case $\varepsilon=1$ we are back to the vacuum case and $\zeta-\varepsilon \zeta_{0}=0$. But if $\varepsilon=\mu=-1$, the NIM case,

$$
z=\hat{\omega}_{ \pm}= \pm \hat{\omega}, \hat{\omega}=\sqrt{\omega_{0}^{2}+\frac{1}{2} \Omega^{2}}
$$

and $\Phi(z)$ can become infinite. Now

$$
\frac{1}{\varepsilon(z)+1}=\frac{1}{2} \frac{z^{2}-\omega_{0}^{2}}{(z-\hat{\omega})(z+\hat{\omega})}, \quad \Phi(z)=\frac{\zeta-\varepsilon \zeta_{0}}{(z-\hat{\omega})(z+\hat{\omega})} \frac{z^{2}-\omega_{0}^{2}}{2 \kappa^{2}\{\varepsilon(z)-1\}},
$$

so we encounter poles in $z= \pm \hat{\omega}$. Next we study the behavior of $\Phi(z)=\Phi(\omega+i \delta)$ as $\delta \downarrow 0$. We start with $\zeta(z)$. In order to obtain the square root in the limit $\delta \downarrow 0$, we must know the signs of $\zeta(\omega)^{2}$ and $\partial_{\omega} \zeta(\omega)^{2}$ in

$$
\zeta(\omega+i \delta)^{2}=\zeta(\omega)^{2}+i \delta \partial_{\omega} \zeta(\omega)^{2}+\mathcal{O}\left(\delta^{2}\right)
$$

where

$$
\zeta(z)^{2}=z^{2}\left(1-\frac{\Omega^{2}}{z^{2}-\omega_{0}^{2}}\right)^{2}-\kappa^{2}, \quad \partial_{z} \zeta(z)^{2}=2 z\left(1-\frac{\Omega^{2}}{z^{2}-\omega_{0}^{2}}\right)\left(1+\frac{\Omega^{2}}{z^{2}-\omega_{0}^{2}}+\frac{2 \omega_{0}^{2} \Omega^{2}}{\left(z^{2}-\omega_{0}^{2}\right)^{2}}\right) .
$$

Since both quantities have definite parity it suffices to consider the case $\omega \geq 0$. We note that $\zeta(0)^{2}=-\kappa^{2}$ and that $\zeta(\omega)^{2}$ increases to $+\infty$ as $\omega$ approaches $\omega_{0}$. Then it decreases again to reach the value $-\kappa^{2}$ for $\omega=\widetilde{\omega}=\sqrt{\omega_{0}^{2}+\Omega^{2}}$. Beyond this value it increases again to tend to $+\infty$ as $\omega \rightarrow+\infty$. Thus $\zeta(\omega)^{2}$ has three zero's, $\omega_{a} \in\left(0, \omega_{0}\right), \omega_{b} \in\left(\omega_{0}, \widetilde{\omega}\right)$, and $\omega_{c}>\widetilde{\omega}$. Since $\zeta(\omega)^{2}=0$ corresponds to a third order equation in $\omega^{2}$, these are the full set of zero's. $\partial_{\omega} \zeta(\omega)^{2}$ vanishes in $\omega=0$, then tends to $+\infty$ as $\omega$ reaches $\omega_{0}$, where it switches sign and increases to 0 in $\widetilde{\omega}$, whereupon it remains positive and eventually tends to $+\infty$. Denoting 


$$
\rho(\omega)=\sqrt{\left|\omega^{2}\left(1-\frac{\Omega^{2}}{\omega^{2}-\omega_{0}^{2}}\right)^{2}-\kappa^{2}\right|},
$$

we obtain (+ indicates that a quantity is positive and - that it is negative)

$\begin{array}{lllllll}\omega \in & >+\omega_{c} & \left(+\widetilde{\omega},+\omega_{c}\right) & \left(+\omega_{b},+\widetilde{\omega}\right) & \left(+\omega_{0},+\omega_{b}\right) & \left(+\omega_{a},+\omega_{0}\right) & \left(0,+\omega_{a}\right) \\ \zeta(\kappa, \omega)^{2} & + & - & - & + & + & - \\ \operatorname{Im} \zeta(\kappa, \omega+i \delta)^{2} & + & + & - & - & + & + \\ \zeta(\kappa, \omega) & \rho(\omega) & i \rho(\omega) & i \rho(\omega) & -\rho(\omega) & \rho(\omega) & i \rho(\omega) \\ & & & & & & \left(-\omega_{a}, 0\right) \\ \omega \in & <-\omega_{c} & \left(-\omega_{c},-\widetilde{\omega}\right) & \left(-\widetilde{\omega},-\omega_{b}\right) & \left(-\omega_{b},-\omega_{0}\right) & \left(-\omega_{0},-\omega_{a}\right) & - \\ \zeta(\kappa, \omega)^{2} & + & - & - & + & + & - \\ \operatorname{Im} \zeta(\kappa, \omega+i \delta)^{2} & - & - & + & + & - & i \rho(\omega) \\ \zeta(\kappa, \omega) & -\rho(\omega) & i \rho(\omega) & i \rho(\omega) & \rho(\omega) & -\rho(\omega) & \end{array}$

In comparison, with $\rho_{0}(\omega)=\sqrt{\left|\omega^{2}-\kappa^{2}\right|}$, so $\rho(\hat{\omega})=\rho_{0}(\hat{\omega})$,

$$
\begin{array}{lllll}
\omega \in & >\kappa & (0,+\kappa) & (-\kappa, 0) & <-\kappa \\
\zeta_{0}(\kappa, \omega) & \rho_{0}(\omega) & i \rho_{0}(\omega) & i \rho_{0}(\omega) & -\rho_{0}(\omega)
\end{array}
$$

Since $\hat{\omega}=\sqrt{\omega_{0}^{2}+\left(\frac{1}{2}\right) \Omega^{2}}$ we have $\hat{\omega} \in\left(\omega_{0}, \widetilde{\omega}\right)$. For $\hat{\omega}>\kappa, \zeta(\kappa, \hat{\omega})^{2}=\hat{\omega}^{2}-\kappa^{2}>0$ and $\hat{\omega} \in\left(\omega_{0}, \omega_{b}\right)$, $\zeta(\kappa, \hat{\omega})=-\rho(\hat{\omega})=-\sqrt{\hat{\omega}^{2}-\kappa^{2}}$, whereas for $\hat{\omega}<\kappa, \zeta(\kappa, \hat{\omega})^{2}<0$ and $\hat{\omega} \in\left(\omega_{b}, \widetilde{\omega}\right), \zeta(\kappa, \hat{\omega})=i \rho(\hat{\omega})$. Similar results follow for $-\hat{\omega}$ resulting in

$$
\begin{array}{lll}
\hat{\omega} \in & >\kappa & (0,+\kappa) \\
\zeta(\kappa,+\hat{\omega}) & -\rho(\hat{\omega}) & i \rho(\hat{\omega}) \\
\zeta(\kappa,-\hat{\omega}) & +\rho(\hat{\omega}) & i \rho(\hat{\omega}) \\
\zeta_{0}(\kappa,+\hat{\omega}) & +\rho(\hat{\omega}) & i \rho(\hat{\omega}) \\
\zeta_{0}(\kappa,-\hat{\omega}) & -\rho(\hat{\omega}) & i \rho(\hat{\omega}) \\
\zeta_{-}(\hat{\omega})-\varepsilon(\hat{\omega}) \zeta_{0}(\hat{\omega}) & 0 & 2 i \rho(\hat{\omega}) \\
\zeta(-\hat{\omega})-\varepsilon(-\hat{\omega}) \zeta_{0}(-\hat{\omega}) & 0 & 2 i \rho(\hat{\omega}) \\
\zeta(\hat{\omega})+\varepsilon(\hat{\omega}) \zeta_{0}(\hat{\omega}) & -2 \rho(\hat{\omega}) & 0 \\
\zeta(-\hat{\omega})+\varepsilon(-\hat{\omega}) \zeta_{0}(-\hat{\omega}) & +2 \rho(\hat{\omega}) & 0
\end{array}
$$

For $\hat{\omega}>\kappa$ and $y>0$ we obtain

$$
\begin{gathered}
G_{p}(x, y, \pm \hat{\omega})= \pm \frac{\rho(\hat{\omega})}{2 i \hat{\omega}^{2}}\{\exp [ \pm i \rho(\hat{\omega})|x-y|] \theta(x)+\exp [ \pm i \rho(\hat{\omega})(x+y)] \theta(-x)\} \\
G_{s}(x, y, \pm \hat{\omega})= \pm \frac{1}{2 i \rho(\hat{\omega})}\{\exp [ \pm i \rho(\hat{\omega})|x-y|] \theta(x)+\exp [ \pm i \rho(\hat{\omega})(x+y)] \theta(-x)\}
\end{gathered}
$$

On the other hand, for $\hat{\omega}<\kappa, \Phi(z)$ becomes infinite for $z= \pm \hat{\omega}$ since $\zeta(\kappa, z)+\varepsilon(z) \zeta_{0}(\kappa, z)$ vanishes in $\pm \hat{\omega}$. Now

$$
\Phi(z)=\frac{\zeta-\varepsilon \zeta_{0}}{(z-\hat{\omega})(z+\hat{\omega})} \frac{z^{2}-\omega_{0}^{2}}{2 \kappa^{2}\{\varepsilon(z)-1\}} \stackrel{z \rightarrow \pm \hat{\omega}}{\sim} \frac{\rho(\hat{\omega}) \Omega^{2}}{4 i \kappa^{2}} \frac{1}{(z-\hat{\omega})(z+\hat{\omega})}
$$

so we encounter poles in $\pm \hat{\omega}$. Thus 


$$
\begin{aligned}
G_{p}(x, y, z)= & K_{0}\left\{\exp \left[i \zeta_{0}|x-y|\right]+\frac{\zeta-\varepsilon \zeta_{0}}{\zeta+\varepsilon \zeta_{0}} \exp \left[i \zeta_{0}(x+y)\right]\right\} \theta(x)+\frac{1}{i z^{2}} \frac{\zeta_{0} \zeta}{\zeta+\varepsilon \zeta_{0}} \exp \left[-i \zeta x+i \zeta_{0} y\right] \theta(-x) \\
= & K_{0} \exp \left[i \zeta_{0}|x-y|\right] \theta(x)+\frac{1}{\zeta+\varepsilon \zeta_{0}}\left\{\kappa_{0}\left(\zeta-\varepsilon \zeta_{0}\right) \exp \left[i \zeta_{0}(x+y)\right] \theta(x)\right. \\
& \left.+\frac{1}{i z^{2}} \zeta_{0} \zeta \exp \left[-i \zeta x+i \zeta_{0} y\right] \theta(-x)\right\} \\
\sim & \frac{\rho(\hat{\omega})}{2 \hat{\omega}^{2}} \exp [-\rho(\hat{\omega})|x-y|] \theta(x)+\frac{\rho(\hat{\omega})^{3} \Omega^{2}}{4 \hat{\omega}^{2} \kappa^{2}} \frac{1}{(z-\hat{\omega})(z+\hat{\omega})}\{\exp [-\rho(\hat{\omega})(x+y)] \theta(x) \\
& +\exp [+\rho(\hat{\omega})(x-y)] \theta(-x)\}, \\
G_{s}(x, y, z)= & L_{0}\left\{\exp \left[i \zeta_{0}|x-y|\right]-\frac{\zeta-\mu \zeta_{0}}{\zeta+\mu \zeta_{0}} \exp \left[i \zeta_{0}(x+y)\right]\right\} \theta(x)-i \frac{\mu}{\zeta+\mu \zeta_{0}} \exp \left[-i \zeta x+i \zeta_{0} y\right] \theta(-x) \\
= & L_{0} \exp \left[i \zeta_{0}|x-y|\right] \theta(x)-\frac{1}{\zeta+\mu \zeta_{0}}\left\{\lambda_{0}\left(\zeta-\mu \zeta_{0}\right) \exp \left[i \zeta_{0}(x+y)\right] \theta(x)+i \mu \exp [-i \zeta x\right. \\
& \left.\left.+i \zeta_{0} y\right] \theta(-x)\right\} \\
& z \rightarrow \pm \hat{\omega} \\
& \sim \frac{1}{2 \rho(\hat{\omega})} \exp [-\rho(\hat{\omega})|x-y|] \theta(x)+i \frac{\rho(\hat{\omega}) \Omega^{2}}{4 i \kappa^{2}} \frac{1}{(z-\hat{\omega})(z+\hat{\omega})}\{\exp [-\rho(\hat{\omega})(x+y)] \theta(x) \\
& +\exp [+\rho(\hat{\omega})(x-y)] \theta(-x)\} .
\end{aligned}
$$

Hence, in the reflection case $x, y>0$, and for $\hat{\omega}>\kappa$,

$$
G_{p}(x, y, \pm \hat{\omega})= \pm \frac{\rho(\hat{\omega})}{2 i z^{2}}\left\{\exp [ \pm i \rho(\hat{\omega})|x-y|], G_{s}(x, y, \pm \hat{\omega})= \pm \frac{1}{2 i \rho(\hat{\omega})}\{\exp [ \pm i \rho(\hat{\omega})|x-y|]\right.
$$

where the term responsible for reflection is absent, i.e., there is no reflection at the frequencies $\pm \hat{\omega}$ for which $\varepsilon(z)=\mu(z)=-1$.

On the other hand, for $\hat{\omega}<\kappa, x, y>0$,

$$
\begin{gathered}
G_{p}(x, y, z) \stackrel{z \rightarrow \pm \hat{\omega}}{\sim} \frac{\rho(\hat{\omega})}{2 \hat{\omega}^{2}} \exp [-\rho(\hat{\omega})|x-y|]+\frac{\rho(\hat{\omega})^{3} \Omega^{2}}{4 \hat{\omega}^{2} \kappa^{2}} \frac{1}{(z-\hat{\omega})(z+\hat{\omega})} \exp [-\rho(\hat{\omega})(x+y)], \\
G_{s}(x, y, z) \stackrel{z \rightarrow \pm \hat{\omega}}{\sim}-\frac{1}{2 \rho(\hat{\omega})} \exp [-\rho(\hat{\omega})|x-y|]+\frac{\rho(\hat{\omega}) \Omega^{2}}{4 \kappa^{2}} \frac{1}{(z-\hat{\omega})(z+\hat{\omega})} \exp [-\rho(\hat{\omega})(x+y)],
\end{gathered}
$$

so now the reflection term is still present but we encounter the damped behavior, typical for the evanescent situation. Next we consider refraction (transmission into the lower half space). Here $y>0>x$, and for $\hat{\omega}>\kappa$,

$$
G_{p}(x, y, \pm \hat{\omega})= \pm \frac{\rho(\hat{\omega})}{2 i \hat{\omega}^{2}} \exp [ \pm i \rho(\hat{\omega})(x+y)], G_{s}(x, y, \pm \hat{\omega})= \pm \frac{1}{2 i \rho(\hat{\omega})} \exp [ \pm i \rho(\hat{\omega})(x+y)]
$$

Now 


$$
\frac{i \kappa}{\zeta(\hat{\omega})^{2}} \partial_{x} G_{p}(x, y, z)=\mp \frac{\kappa}{\rho(\hat{\omega})},
$$

leading to

$$
\begin{aligned}
\mathbf{G}_{\boldsymbol{\kappa}}(x, y, z) \stackrel{z \rightarrow \pm \hat{\omega}}{\rightarrow} & \pm \frac{1}{2 i \rho(\hat{\omega})} \exp [ \pm i \rho(\hat{\omega})(y+x)] \\
& \times\left\{\frac{\rho(\hat{\omega})^{2}}{\hat{\omega}^{2}}\left(\mathbf{e}_{\boldsymbol{\kappa}} \mp \frac{\kappa}{\rho(\hat{\omega})} \mathbf{e}_{3}\right)\left(\mathbf{e}_{\boldsymbol{\kappa}} \mp \frac{\kappa}{\rho(\hat{\omega})} \mathbf{e}_{3}\right)+\mathbf{e}_{3} \times \mathbf{e}_{\boldsymbol{\kappa}} \mathbf{e}_{3} \times \mathbf{e}_{\boldsymbol{\kappa}}\right\},
\end{aligned}
$$

whereas in the vacuum case,

$$
\begin{aligned}
\mathrm{G}_{\boldsymbol{\kappa}}(x, y, \pm \hat{\omega})= & \pm \frac{1}{2 i \rho(\hat{\omega})} \exp [ \pm i \rho(\hat{\omega})(y-x)] \\
& \times\left\{\frac{\rho(\hat{\omega})^{2}}{\hat{\omega}^{2}}\left(\mathbf{e}_{\boldsymbol{\kappa}} \pm \frac{\kappa}{\rho(\hat{\omega})} \mathbf{e}_{3}\right)\left(\mathbf{e}_{\boldsymbol{\kappa}} \pm \frac{\kappa}{\rho(\hat{\omega})} \mathbf{e}_{3}\right)+\mathbf{e}_{3} \times \mathbf{e}_{\boldsymbol{\kappa}} \mathbf{e}_{3} \times \mathbf{e}_{\boldsymbol{\kappa}}\right\}
\end{aligned}
$$

Comparing the two we note that $x$ has changed to $-x$ and $\kappa / \rho(\hat{\omega})$ to $-\kappa / \rho(\hat{\omega})$, showing the anomalous behavior found earlier for NIM systems. This behavior becomes more direct in a scattering formalism where it would show up in the corresponding scattering amplitude. However, setting up a scattering formalism, although a straightforward matter using the auxiliary field approach (the dielectric case was treated earlier in Ref. 6), involves a substantial amount of bookkeeping. This is mainly due to the existence of two different scattering channels for reflection and transmission. The scattering situation is somewhat less complicated for a single NIM layer, where the transmitted wave eventually is moving in vacuum again.

If $\hat{\omega}<\kappa$, for $y>0>x$,

$$
G_{p}(x, y, z) \stackrel{z \rightarrow \pm \hat{\omega}}{\sim} \frac{\rho(\hat{\omega})^{3} \Omega^{2}}{4 \hat{\omega}^{2} \kappa^{2}\left(z^{2}-\hat{\omega}^{2}\right)} \exp [-\rho(\hat{\omega})(y-x)], G_{s}(x, y, z) \stackrel{z \rightarrow \pm \hat{\omega}}{\sim} \frac{\rho(\hat{\omega}) \Omega^{2}}{4 \kappa^{2}\left(z^{2}-\hat{\omega}^{2}\right)} \exp [-\rho(\hat{\omega})(y-x)],
$$

once more showing evanescent behavior. In retrieving $\boldsymbol{E}(\mathbf{x}, t)$, the pole contributions in Green's function give rise to terms oscillating in time according to $\exp [ \pm i \hat{\omega} t]$, so no damping occurs in the time dependence, a property observed earlier by Pendry ${ }^{2}$ for the case of a single layer.

\section{DISCUSSION}

\section{A. Summary of results}

We started off with a system characterized by general causal, passive, susceptibilities $\chi_{e}(\mathbf{x}, t)$ and $\chi_{m}(\mathbf{x}, t)$ and showed, using the auxiliary field approach, that $\boldsymbol{E}(\mathbf{x}, t)$ and $\boldsymbol{H}(\mathbf{x}, t)$ have a proper time evolution. If they are square integrable at the initial time this remains true at all later times so possible singularities are square integrable and the electromagnetic energy remains finite. We then specialized to layered systems using a Laplace transformed formalism. We expressed Green's function, which is a tensor, in terms of the two scalar functions $G_{s}(x, y, z)$ and $G_{p}(x, y, z)$. A consequence of the auxiliary field setup is that the Helmholtz Green's function is square integrable in both coordinates. In particular, this is true in the evanescent case.

We then studied the special situation where one half space is vacuum and the other filled with a medium. Restricting ourselves to scalar permeabilities given by a single dispersive Lorentz term, 


$$
\varepsilon(z)=\mu(z)=1-\frac{\Omega^{2}}{z^{2}-\omega_{0}^{2}}, \quad \operatorname{Im} z>0,
$$

which take on the value of -1 for $z= \pm \hat{\omega}= \pm\left(\omega_{0}^{2}+\frac{1}{2} \Omega^{2}\right)^{1 / 2}$, we then evaluated $G_{s}(x, y, z)$ and $G_{p}(x, y, z)$ as $z$ approaches these values and found, for the nonevanescent case, a typical NIM behavior, the reflected field vanishes whereas the transmitted field behaves anomalously, in accordance with earlier results on NIM systems. As is well known, although $n^{2}=\varepsilon( \pm \hat{\omega}) \mu( \pm \hat{\omega})=1$, the NIM case is different from the vacuum situation as is seen from the refractive behavior. But the absence of reflection in the radiative regime is shared by both.

On the other hand Green's function has poles at $\pm \hat{\omega}$ in the evanescent situation. They lead to oscillating terms proportional to $\exp [ \pm i \hat{\omega} t]$ and the reflective part of Green's function no longer vanishes. There is no damping in the temporal behavior as noted earlier by Pendry. ${ }^{2}$ This is obvious since the former only occurs in absorptive media, the spatial fall-off occurring in evanescent situations is completely unrelated to the temporal decay found in the absorptive case.

Note that in the present setup the NIM case is a special situation occurring for two discrete frequencies $\pm \hat{\omega}$ among a whole set where no NIM behavior takes place. The general expression for the permeabilities of a dispersive, nonabsorptive, system for equal $\varepsilon$ and $\mu$ is a (possibly infinite) sum of dispersive Lorentz terms,

$$
\varepsilon(z)=\mu(z)=1-\sum_{n} \frac{\Omega_{n}^{2}}{z^{2}-\omega_{0 n}^{2}},
$$

and in this case there is a larger set of frequencies $\hat{\omega}_{n}$ for which $\varepsilon=\mu=-1$. But between these frequencies $\varepsilon$ and $\mu$ vary wildly, in particular, there is always a $\omega_{0 n}$ (for which there is no transmission) between two subsequent $\hat{\omega}_{n}$ 's. This may spoil the idea of obtaining an interval for which this relation is approximately valid. Indeed, if in $z=\omega+i \delta, \omega$ approaches $\omega_{0 n}$, then

$$
\varepsilon(z) \sim \frac{\delta \downarrow \Omega_{n}^{2}}{2 \omega_{0 n}} \frac{1}{\delta}
$$

In the half space case this results in

$$
\frac{1}{\zeta+\varepsilon \zeta_{0}} \stackrel{\delta \downarrow 0}{\rightarrow} 0, \quad \frac{\zeta-\varepsilon \zeta_{0}}{\zeta+\varepsilon \zeta_{0}} \rightarrow-1
$$

so $\mathrm{G}_{\boldsymbol{\kappa}}(x, y, z)$ vanishes in this limit if $x<0$ and $y>0$. Thus the situation is opposite to the NIM case. In that case there is no reflection, whereas here the transmission vanishes (perfect reflector).

We did not consider absorption in the NIM case. This is straightforward to do along the same lines but since the medium extends over a half space, the transmitted field will die out. The two poles $\pm \hat{\omega}$ now acquire a negative imaginary part, so Green's function remains finite for all frequencies and the reflection term in Green's function no longer vanishes. We intend to come back to this situation for the single layer case, where a transmitted field, although attenuated, is still present. Moreover, this case can fairly easily be treated in terms of a scattering formalism, leading to scattering amplitudes for reflection and transmission, which should show NIM behavior in the dispersive case for appropriate frequencies.

\section{B. Discrete eigenvalues of $\mathrm{K}$, surface modes, and radiative atomic decay}

We found earlier that the Helmholtz Green's function of a NIM system had poles in $z= \pm \hat{\omega}$. Usually poles in Green's function originate from discrete eigenvalues of the original operator and this is precisely what happens here. In the more general case that $\lambda_{e} \neq \lambda_{m}$ and $\Omega_{e} \neq \Omega_{m}$, the condition $\varepsilon(z) \mu(z)=1$ again gives the solutions $z= \pm \hat{\omega}$ but now 


$$
\hat{\omega}^{2}=\frac{\lambda_{e}^{2} \Omega_{m}^{2}+\lambda_{m}^{2} \Omega_{e}^{2}+\Omega_{e}^{2} \Omega_{m}^{2}}{\Omega_{e}^{2}+\Omega_{m}^{2}} .
$$

It is straightforward to show that in the half space case $\pm \hat{\omega}$ are eigenvalues of $\mathrm{K}$ with associated eigenfunctions proportional to

$$
\exp \left[i \boldsymbol{\kappa} \cdot \mathbf{x}^{\perp}\right] \exp \left[-\sqrt{\kappa^{2}-\hat{\omega}^{2}}\left|x_{3}\right|\right], \quad \kappa>\hat{\omega},
$$

i.e., surface modes. Since $\hat{\omega}$ does not depend on $\kappa$, a general theorem about direct integral decompositions tells us that $\pm \hat{\omega}$ are discrete eigenvalues of $\mathrm{K}$ with infinite degeneracy. This can also be seen directly since by superposition we can construct an infinite orthonormal set of square integrable functions $\left\{\mathbf{f}_{n}(\mathbf{x})\right\}$,

$$
\mathbf{f}_{n}(\mathbf{x})=\int d \boldsymbol{\kappa} \rho_{n}(\boldsymbol{\kappa}) \exp \left[i \boldsymbol{\kappa} \cdot \mathbf{x}^{\perp}\right] \exp \left[-\sqrt{\kappa^{2}-\hat{\omega}^{2}}\left|x_{3}\right|\right] .
$$

Here the question arises as to what happens if there are whole sets of dispersive Lorentz terms in the susceptibilities. Then there will be sets of solutions $\left\{ \pm \hat{\omega}_{n}\right\}$, but the condition $\kappa>\hat{\omega}$ needs refinement.

Excited atoms in vacuum decay by photon emission providing there is no selection rule forbidding the transition. It is well known that the radiative decay constant $\gamma_{\text {vac }}$ changes if we no longer have vacuum. Examples are atoms in a cavity or near a material such as a dielectric. This is caused by alterations in the field modes relative to the vacuum case. In simple situations, making the dipole and isotropic approximation, $\gamma$ can be expressed in terms of the imaginary part of the trace (Tr) of Green's function as a matrix,

$$
\gamma \sim \operatorname{Im} \operatorname{Tr} \mathrm{G}\left(\mathbf{x}, \mathbf{x}, \omega_{\text {tr }}\right),
$$

where $\mathbf{x}$ is the atomic position and $\omega_{t r}$ the atomic transition frequency. In case the permeabilities are frequency independent, $\operatorname{Im} \operatorname{Tr} G(\mathbf{x}, \mathbf{x}, \omega)$ is the local density of states. In Ref. 6 the above result was obtained for dielectrics. The same procedure, involving quantization of the fields, can be used in the present case with the same result (the case of an atom embedded in a magnetodielectric material was considered by the Jena group ${ }^{9}$ ). Here we make a few remarks about the layered case. We have

$$
\operatorname{Im} \operatorname{Tr} \mathrm{G}\left(\mathbf{x}, \mathbf{x}, \omega_{t r}\right)=\int d \boldsymbol{\kappa} \operatorname{Im} \operatorname{Tr} \mathrm{G}_{\boldsymbol{\kappa}}\left(x, x, \omega_{\mathrm{tr}}\right)
$$

and consider the dispersive half space situation with the atom in vacuum close to the interface. Since Green's function for $x, y>0$ is the sum of a vacuum and reflective part, we can write $\gamma$ $=\gamma_{\text {vac }}+\gamma_{\text {ref. }}$. At the NIM frequency, $\omega_{t r}=\hat{\omega}$, there is no reflection in the propagating regime so in the $\boldsymbol{\kappa}$-integral only $\kappa>\hat{\omega}$ can contribute. Since $\operatorname{Im} \mathrm{G}_{\boldsymbol{\kappa}}\left(x, x, \omega_{t r}+i 0\right)$ becomes infinite for $\omega_{t r}=\hat{\omega}$, due to the presence of the $\delta$-functions $\delta(\omega \pm \hat{\omega})$, we obtain an infinite result for $\gamma_{\text {ref }}$. Such an infinite local density of states was also encountered in Ref. 10, where a so-called perfect corner reflector was considered. However, this result is incorrect. Upon quantization, an excited atom with $\omega_{t r}=\hat{\omega}$ can decay radiatively but also transitions to the above bound states of $\mathrm{K}$ are possible. The latter process is an oscillatory one and we expect decay but modulated by oscillations. However, there are no infinities.

Another point is that the situation is highly anisotropic so the isotropy approximation, which is used to obtain Eq. (7.8), becomes doubtful. Clearly the radiative decay problem needs further study.

\section{Fixed frequency model}

In Sec. I we mentioned that using a simple model with fixed, i.e., frequency independent $\varepsilon$ $=\mu=-1$ can give rise to problems in calculating Green's function. Indeed, our formalism indicates 
that, in the evanescent case, there are poles in $\pm \hat{\omega}$, so Green's function becomes infinite and the simple model breaks down. As we have seen, in retrieving $\boldsymbol{E}(\mathbf{x}, t)$ a finite result emerges and the responsible frequency integration mechanism is absent in the model. In addition there is a second flaw. With

$$
\varepsilon=\mu=\varepsilon\left(x_{3}\right)=\mu\left(x_{3}\right)= \begin{cases}+1, & x_{3}>0 \\ -1, & x_{3}<0,\end{cases}
$$

the conserved energy

$$
\mathcal{E}=\frac{1}{2} \int d \mathbf{x}\left\{\varepsilon\left(x_{3}\right) \boldsymbol{E}(\mathbf{x}, t)^{2}+\mu\left(x_{3}\right) \boldsymbol{B}(\mathbf{x}, t)^{2}\right\}
$$

is no longer positive definite and we can no longer base an inner product and associated Hilbert space formalism on this quantity. We still can use the inner product,

$$
(\mathbf{f}, \mathbf{g})=\int d \mathbf{x} \mathbf{f}(\mathbf{x}) \cdot \overline{\mathbf{g}(\mathbf{x})},
$$

but now the candidate for the generator of the time evolution, determined by the usual boundary conditions, is no longer self-adjoint, so the existence of a time evolution comes into question. Of course it is possible to introduce a Krein space with inner product based on $\mathcal{E}$ but this does not solve this problem. An alternative is to restrict $K$ to the eigenspaces associated with $\pm \hat{\omega}$, which leads to a correct time evolution. Then we have a fixed frequency model but now the part of $\mathrm{K}$, relevant for radiative decay, is missing.

\section{Response to an external source}

We consider the time evolution due to an external source. In general, the source is given by charge and current densities $\rho_{\mathrm{ext}}(\mathbf{x}, t)$ and $\boldsymbol{J}_{\mathrm{ext}}(\mathbf{x}, t)$, which are related by the conservation law,

$$
\partial_{t} \rho_{\mathrm{ext}}(\mathbf{x}, t)+\partial_{\mathbf{x}} \cdot \boldsymbol{J}_{\mathrm{ext}}(\mathbf{x}, t)=0 .
$$

We assume that the source quantities vanish for $t \leq t_{0}$, so the same holds for the fields. Then

$$
\partial_{t} \boldsymbol{D}(\mathbf{x}, t)=\partial_{\mathbf{x}} \times \boldsymbol{H}(\mathbf{x}, t)-\boldsymbol{J}_{\mathrm{ext}}(\mathbf{x}, t), \quad \partial_{t} \boldsymbol{F}(t)=-i \mathrm{~K} \cdot \boldsymbol{F}(t)-\boldsymbol{G}(t),
$$

where $\boldsymbol{G}_{1}(t)=\boldsymbol{J}_{\text {ext }}(\mathbf{x}, t)$, whereas its other components vanish. Since $\boldsymbol{F}\left(t_{0}\right)=0$, Duhamel's formula gives

$$
\boldsymbol{F}(t)=\int_{t_{0}}^{t} d s \exp [-i \mathrm{~K}(t-s)] \cdot \boldsymbol{G}(s) .
$$

We are interested in the behavior of $\boldsymbol{F}(t)$ for large $t$. This depends on the nature of the spectrum of $\mathrm{K}$. We assume that $\mathrm{K}$ does not have singular continuous spectrum, so

$$
\mathrm{K}=\sum_{n} \lambda_{n} \mathrm{P}_{n}+\int \lambda \boldsymbol{E}_{a c}(d \lambda)=\sum_{n} \lambda_{n} \mathrm{P}_{n}+\sum_{\alpha} \int d \lambda \lambda\left|\mathbf{u}_{\lambda \alpha}\right\rangle\left\langle\mathbf{u}_{\lambda \alpha}\right| .
$$

At this point we set $t_{0}=0$ and assume that

$$
\boldsymbol{G}(t)=f(t) \boldsymbol{G}_{0}, \quad \boldsymbol{G}_{0} \in \mathcal{K},
$$

where the Fourier transform $\widetilde{f}(\omega)$ in 


$$
f(t)=\int d \omega \exp [-i \omega t] \tilde{f}(\omega)
$$

is a smooth function of $\omega$. Then

$$
\begin{gathered}
\mathrm{P}_{n} \cdot \boldsymbol{F}(t)=\int_{0}^{t} d s \exp \left[-i \lambda_{n}(t-s)\right] f(s) \mathrm{P}_{n} \cdot \boldsymbol{G}_{0}, \\
\mathbf{P}_{a c} \cdot \boldsymbol{F}(t)=\int_{0}^{t} d s \sum_{\alpha} \int d \lambda \exp [-i \lambda(t-s)] f(s)\left|\mathbf{u}_{\lambda \alpha}\right\rangle\left\langle\mathbf{u}_{\lambda \alpha} \mid \boldsymbol{G}_{0}\right\rangle, \\
\left\langle\mathbf{u}_{\lambda \alpha} \mid \mathrm{P}_{a c} \cdot \boldsymbol{F}(t)\right\rangle=\int_{0}^{t} d s \exp [-i \lambda(t-s)] f(s)\left\langle\mathbf{u}_{\lambda \alpha} \mid \boldsymbol{G}_{0}\right\rangle,
\end{gathered}
$$

and

$$
\begin{aligned}
\mathrm{P}_{n} \cdot \boldsymbol{F}(t)= & \int_{0}^{t} d s \exp \left[-i \lambda_{n}(t-s)\right] \mathrm{P}_{n} \cdot \int d \omega \exp [-i \omega s] \widetilde{\boldsymbol{G}}(\omega) \\
& =\exp \left[-i \lambda_{n} t\right] \int d \omega \frac{\exp \left[i\left(\lambda_{n}-\omega\right) t\right]-1}{i\left(\lambda_{n}-\omega\right)} \widetilde{f}(\omega) \mathrm{P}_{n} \cdot \boldsymbol{G}_{0} \\
& =\exp \left[-i \lambda_{n} t\right] \int d v \frac{1-\exp [-i v]}{-i v} \widetilde{f}\left(\lambda_{n}+\frac{v}{t}\right) \mathrm{P}_{n} \cdot \boldsymbol{G}_{0} \\
& \stackrel{t \rightarrow \infty}{ }+\frac{\pi}{2} \exp \left[-i \lambda_{n} t\right] \tilde{f}\left(\lambda_{n}\right) \mathrm{P}_{n} \cdot \boldsymbol{G}_{0},
\end{aligned}
$$

whereas

$$
\left\langle\mathbf{u}_{\lambda \alpha} \mid \mathrm{P}_{a c} \cdot \boldsymbol{F}(t)\right\rangle^{t \rightarrow \infty} \sim \frac{\pi}{2} \exp [-i \lambda t] \widetilde{f}(\lambda)\left\langle\mathbf{u}_{\lambda \alpha} \mid \boldsymbol{G}_{0}\right\rangle,\left\|\mathrm{P}_{a c} \cdot \boldsymbol{F}(t)\right\|^{2 \rightarrow \infty} \rightarrow\left(\frac{\pi}{2}\right)^{2} \sum_{\alpha} \int d \lambda|\widetilde{f}(\lambda)|^{2}\left|\left\langle\mathbf{u}_{\lambda \alpha} \mid \boldsymbol{G}_{0}\right\rangle\right|^{2}
$$

We see that for large times $\mathrm{P}_{n} \cdot \boldsymbol{F}(t)$ oscillates at the frequency $\omega_{n}$, a familiar situation for musical instruments excited by a pulse (playing a piano, ringing a bell). We have seen that in the dispersive half space case $\mathrm{K}$ has eigenvalues $\pm \hat{\boldsymbol{\omega}}$. Hence $\boldsymbol{E}(\mathbf{x}, t)$ has contributions that oscillate at these frequencies. Whether or not other contributions eventually die out depends on the nature of $\tilde{f}(\lambda)$ and $\left\langle\mathbf{u}_{\lambda \alpha} \mid \boldsymbol{G}_{0}\right\rangle$. If the two have disjoint supports in $\lambda$, this will indeed be the case. However, the continuous spectrum of $\mathrm{K}$ typically covers the whole real axis.

Actual sources always have a finite bandwidth (although it can be quite small as for single mode laser sources). In practice monochromatic sources (which are usually simplified to a point source) are often considered. Thus if, for instance, $f(t)=f_{0} \sin \omega_{0} t$, then $\tilde{f}(\lambda)=(2 i)^{-1} f_{0}\left\{\delta\left(\lambda-\omega_{0}\right)\right.$ $\left.-\delta\left(\omega+\omega_{0}\right)\right\}$. Now $\mathrm{P}_{n} \cdot \boldsymbol{F}(t)$ vanishes if $\lambda_{n} \neq \pm \omega_{0}$ and diverges for $\lambda_{n}= \pm \omega_{0}$. Also

$$
\left\langle\mathbf{u}_{\lambda \alpha} \mid \mathrm{P}_{a c} \cdot \boldsymbol{F}(t)\right\rangle=\frac{f_{0}}{2 i} \exp [-i \lambda t]\left\{\frac{\exp \left[i\left(\lambda-\omega_{0}\right) t\right]-1}{i\left(\lambda-\omega_{0}\right)}-\frac{\exp \left[i\left(\lambda+\omega_{0}\right) t\right]-1}{i\left(\lambda+\omega_{0}\right)}\right\}\left\langle\mathbf{u}_{\lambda \alpha} \mid \boldsymbol{G}_{0}\right\rangle,
$$

and $\mathrm{P}_{a c} \cdot \boldsymbol{F}(t)$ diverges unless $\left\langle\mathbf{u}_{\lambda, \alpha} \mid \boldsymbol{G}_{0}\right\rangle$ vanishes in a neighborhood of $\pm \omega_{0}$. Such divergent behavior is typical for undamped systems driven by a harmonic force. The external source situation with a monochromatic point source is sometimes used as the starting point for the calculation 
of Green's functions. Then, with $\boldsymbol{G}(\mathbf{x}, t)=\boldsymbol{G}_{0} \exp \left[-i \omega_{0} t\right] \delta\left(\mathbf{x}-\mathbf{x}_{0}\right)$, taking Fourier transforms,

$$
[\omega-\mathrm{K}] \cdot \tilde{\boldsymbol{F}}(\omega)=i \delta\left(\omega-\omega_{0}\right) \delta\left(\mathbf{x}-\mathbf{x}_{0}\right) \boldsymbol{G}_{0} .
$$

However, $\omega-\mathrm{K}$ does not have an inverse, $\omega$ being in the spectrum of $\mathrm{K}$. This problem is avoided by using Laplace transforms, in which case

$$
\hat{\boldsymbol{F}}(z)=i[z-\mathrm{K}]^{-1} \cdot \hat{\boldsymbol{G}}(z), \quad \operatorname{Im} z>0 .
$$

Returning to the half space case, we conclude that for large times $\boldsymbol{E}(\mathbf{x}, t)$ has oscillating contributions at the frequencies $\pm \hat{\omega}$ but that there is also an additional contribution associated with the continuous spectrum of $\mathrm{K}$.

\section{E. Final remarks}

The philosophy of our approach is to study special properties that occur for specific frequencies, the NIM case being the primary example. But, as already noted, in the half space case with a single scalar Lorentz contribution, transmission tends to 0 if $\omega \rightarrow \omega_{0}$. For this to happen, $\varepsilon(z)$ and $\mu(z)$ need not be identical, as can be seen from the expression for Green's function. This feature remains valid if there are more Lorentz contributions present. It suggests the experimental study of reflection properties as a function of frequency. Dips will occur if a NIM situation can happen (absorption will prevent obtaining an exact zero) and maxima at the frequencies $\omega_{0 n}$ in Eq. (7.2). In the idealized single Lorentz case $\omega_{0}$ and $\hat{\omega}$ can both be obtained in this way.

Although we concentrated on the situation where the electric and magnetic susceptibilities were given by the same single dispersive Lorentz form, the situation where they consist of different sets of Lorentz contributions can also give rise to NIM situations, the fundamental requirement being the existence of some frequencies $\hat{\omega}$ for which $\varepsilon(\hat{\omega})=\mu(\hat{\omega})=-1$.

An important question is in how far such systems can be realized. On a microscopic level it seems not to be possible to obtain this property (for some further considerations on the susceptibilities of magnetodielectric systems, see Ref. 11). On a larger scale, small structures, involving split rings and other configurations, lead to a more favorable situation. But if the electric and magnetic modes are coupled, complications arise (von Neumann's noncrossing rule). Another point is that, due to the employed manufacturing methods, fabricated materials are not isotropic. But this may change in the future. There is an extensive experimental literature concerning the fabrication of such devices. ${ }^{5} \mathrm{~A}$ further complication is often the occurrence of losses. The latter can spoil the delicate effects essential for "perfect lenses." In principle such lenses can consist of a single NIM slab. In the present work we did not consider this case, a simple form of a layered system. However, in a quite recent publication, Collin ${ }^{12}$ made a precise analysis of this specific case. He took into account field contributions not considered by Pendry ${ }^{2}$ and the effect of fields switched on for only a finite time interval and found that taking these into account spoils the perfect lens behavior. We also encountered such contributions in the half space case, the background part in addition to the pole terms in Green's functions.

Losses will blur the NIM behavior originating from pole contributions in a dispersive case, since the poles now acquire an imaginary part. This raises the question if adding some gain can improve the situation. Typically losses arise from a coupling of the electromagnetic field to material modes that have continuous spectrum. As an example, if the material consists of a single atom, this coupling is the one to atomic continuum states (ionization). In macroscopic media, among other possibilities, couplings to phonon modes and the occurrence of Förster processes can cause absorption. In such situations electromagnetic energy is converted to material modes where the energy leaks away in space. It will be clear that compensating all losses by means of adding gain (this would convert the system to a dispersive, nonabsorptive one) will not be possible. But compensating loss at a few specific frequencies, for instance, by pumping the system to create level inversions in the material subsystem, may be feasible. Here the gain must be controlled precisely in order to maintain passivity and avoid undesirable instabilities. This situation was 
analyzed by Stockman, ${ }^{4,13}$ who applied a causality argument to the square of the refraction function $n(z)^{2}=\varepsilon(z) \mu(z)$. He concluded that negative refraction cannot be accomplished by adding gain.

Finally, we mention an approach based on space-time transformations leading to a simpler set of field equations but in a curved space-time frame. ${ }^{14}$ In particular, the detailed work by Leonhardt and Philbin, using methods borrowed from general relativity, should be mentioned. So far this approach is restricted to frequency independent $\varepsilon$ and $\mu$. Taking the AFF as a starting point it may be possible to extend it to the general frequency-dependent case.

\section{ACKNOWLEDGMENTS}

The work of B.G. was partly supported by the project FANI (Grant No. ANR-07-NANO-03803) of the program PNANO funded by the Agence Nationale de la Recherche. A.T. was supported by FOM with financial support by NWO.

\section{APPENDIX A: SELF-ADJOINTNESS OF $K$}

The idea is to split $\mathrm{K}$ into a zero order part $\mathrm{K}_{0}$ and a perturbation $\mathrm{K}_{1}$,

$$
\mathrm{K}=\mathrm{K}_{0}+\mathrm{K}_{1} \text {, }
$$

where

$$
\mathrm{K}_{0}=\left(\begin{array}{cccccc}
0 & 0 & 0 & \boldsymbol{\epsilon} \cdot \mathbf{p} & 0 & 0 \\
0 & 0 & 0 & 0 & 0 & i \lambda \\
0 & 0 & 0 & 0 & -i \lambda & 0 \\
-\boldsymbol{\epsilon} \cdot \mathbf{p} & 0 & 0 & 0 & 0 & 0 \\
0 & 0 & i \lambda & 0 & 0 & 0 \\
0 & -i \lambda & 0 & 0 & 0 & 0
\end{array}\right), \quad \mathrm{K}_{1}=\left(\begin{array}{cccccc}
0 & 0 & 0 & 0 & 0 & -i\left\langle\boldsymbol{\nu}_{e}\right| \\
0 & 0 & 0 & 0 & 0 & 0 \\
0 & 0 & 0 & i & 0 & 0 \\
0 & 0 & -i\left\langle\boldsymbol{\nu}_{m}\right| & 0 & 0 & 0 \\
0 & 0 & 0 & 0 & 0 & 0 \\
i & 0 & 0 & 0 & 0 & 0
\end{array}\right) .
$$

Proposition: Assume that $\left\|\boldsymbol{\chi}_{e, m}^{\prime}(\mathbf{x}, 0)\right\|_{\infty}=\sup _{\mathbf{x}}\left|\boldsymbol{\chi}_{e, m}^{\prime}(\mathbf{x}, 0)\right| \leq c<\infty$. Then $\mathrm{K}_{1}$ is bounded so $\mathrm{K}$ is selfadjoint with domain $\mathcal{D}\left(\mathrm{K}_{0}\right)$.

Proof: For notational simplicity we give the proof for scalar susceptibilities and the absorptive case [so $\nu_{e, m}(\mathbf{x}, \lambda)^{1 / 2}$ are properly defined, the dispersive situation must be handled slightly differently]. Let $\mathbf{f} \in \mathcal{D}\left(\mathrm{K}_{0}\right)$. Then

$$
\mathbf{g}=\left(\begin{array}{l}
\mathbf{g}_{1} \\
\mathbf{g}_{2} \\
\mathbf{g}_{3} \\
\mathbf{g}_{4} \\
\mathbf{g}_{5} \\
\mathbf{g}_{6}
\end{array}\right)=\mathrm{K}_{1} \cdot \mathbf{f}=\left(\begin{array}{c}
-i \int d \lambda \nu_{e}(\mathbf{x}, \lambda) \mathbf{f}_{6}(\mathbf{x}, \lambda) \\
0 \\
i \mathbf{f}_{4}(\mathbf{x}) \\
-i \int d \lambda \nu_{m}(\mathbf{x}, \lambda) \mathbf{f}_{3}(\mathbf{x}, \lambda) \\
0 \\
i \mathbf{f}_{1}(\mathbf{x})
\end{array}\right)
$$

Now 


$$
\begin{aligned}
\left\|\mathbf{g}_{1}\right\|_{1}^{2}= & \int d \mathbf{x} \int d \lambda \nu_{e}(\mathbf{x}, \lambda) \mathbf{f}_{6}(\mathbf{x}, \lambda) \cdot \int d \mu \nu_{e}(\mathbf{x}, \mu) \overline{\mathbf{f}_{6}(\mathbf{x}, \mu)} \\
= & \int d \mathbf{x} \int d \lambda \nu_{e}(\mathbf{x}, \lambda)^{1 / 2} \int d \mu \nu_{e}(\mathbf{x}, \mu)^{1 / 2} \nu_{e}(\mathbf{x}, \lambda)^{1 / 2} \mathbf{f}_{6}(\mathbf{x}, \lambda) \cdot \nu_{e}(\mathbf{x}, \mu)^{1 / 2} \overline{\mathbf{f}_{6}(\mathbf{x}, \mu)} \\
& \leq \int d \mathbf{x}\left[\int d \lambda \nu_{e}(\mathbf{x}, \lambda)\right]^{1 / 2}\left[\int d \mu \nu_{e}(\mathbf{x}, \mu)\right]^{1 / 2} \\
& \times\left[\int d \lambda \nu_{e}(\mathbf{x}, \lambda)\left|\mathbf{f}_{6}(\mathbf{x}, \lambda)\right|^{2}\right]^{1 / 2}\left[\int d \mu \nu_{e}(\mathbf{x}, \mu)\left|\mathbf{f}_{6}(\mathbf{x}, \mu)\right|^{2}\right]^{1 / 2} \\
= & \int d \mathbf{x} \int d \lambda \nu_{e}(\mathbf{x}, \lambda) \int d \lambda \nu_{e}(\mathbf{x}, \lambda)\left|\mathbf{f}_{6}(\mathbf{x}, \lambda)\right|^{2}=\int d \mathbf{x} \chi^{\prime}(\mathbf{x}, 0) \int d \lambda \nu_{e}(\mathbf{x}, \lambda)\left|\mathbf{f}_{6}(\mathbf{x}, \lambda)\right|^{2} \\
& \leq\left\|\chi^{\prime}(0)\right\|_{\infty}\left\|\mathbf{f}_{6}\right\|_{6}^{2} \leq d\left\|\mathbf{f}_{6}\right\|_{6}^{2} .
\end{aligned}
$$

Also

$$
\left\|\mathbf{g}_{4}\right\|_{3}^{2}=\int d \mathbf{x} \int d \lambda \nu_{m}(\mathbf{x}, \lambda)\left|\mathbf{f}_{3}(\mathbf{x})\right|^{2} \leq d\left\|\mathbf{f}_{3}\right\|_{4}^{2}
$$

and similar for the other components. Thus

$$
\left\|\mathbf{K}_{1} \cdot \mathbf{f}\right\| \leq \sqrt{d+1}\|\mathbf{f}\|
$$

so $\mathrm{K}_{1}$ is a bounded self-adjoint operator and hence $\mathrm{K}$ is self-adjoint with domain $\mathcal{D}\left(\mathrm{K}_{0}\right)$.

Remark: Note that the proof does not require $\nu_{e, m}(\mathbf{x}, \lambda)$ to be non-negative or even real. However, if this is not the case the inner product on $\mathcal{K}$ is altered and the norm no longer non-negative.

\section{APPENDIX B: PROJECTIONS OF R(z)}

We assume the susceptibilities to be scalar and consider $P_{1}[z-K]^{-1} P_{1}=P_{1} R(z) P_{1}$, where $P_{j}$ projects upon the $j$ th component of $\mathbf{f} \in \mathcal{K}, \mathrm{P}_{j} \mathbf{f}=\mathbf{f}_{j}$. We have

$$
\begin{aligned}
{[z-\mathrm{K}]^{-1} } & =[z+\mathrm{K}]\left[z^{2}-\mathrm{K}^{2}\right]^{-1} \\
& =[z+\mathrm{K}]\left(\begin{array}{rr}
{\left[z^{2}-\mathrm{K}_{e m} \cdot \mathrm{K}_{m e}\right]^{-1}} & 0 \\
0 & \\
& {\left[z^{2}-\mathrm{K}_{m e} \cdot \mathrm{K}_{e m}\right]^{-1}}
\end{array}\right) \\
& =[z+\mathrm{K}]\left(\begin{array}{cc}
{\left[z^{2}-\mathrm{H}_{e}\right]^{-1}} & 0 \\
0 & {\left[z^{2}-\mathrm{H}_{m}\right]^{-1}}
\end{array}\right), \\
\mathbf{H}_{e} & =\left(\begin{array}{lll}
\mathrm{h}_{0}+\chi_{e}^{\prime}(\mathbf{x}, 0) & -\left\langle\nu_{e}\right| \lambda & -i \boldsymbol{\epsilon} \cdot \mathbf{p}\left\langle\nu_{m}\right| \\
-\lambda & \lambda^{2} & 0 \\
-i \boldsymbol{\epsilon} \cdot \mathbf{p} & 0 & \lambda^{2}+|0\rangle\left\langle\nu_{m}\right|
\end{array}\right), \quad \mathrm{H}_{m}=\left(\begin{array}{lll}
\mathrm{h}_{0}+\chi_{m}^{\prime}(\mathbf{x}, 0) & -\left\langle\nu_{m}\right| \lambda & i \boldsymbol{\epsilon} \cdot \mathbf{p}\left\langle\nu_{e}\right| \\
-\lambda & \lambda^{2} & 0 \\
i \boldsymbol{\epsilon} \cdot \mathbf{p} & 0 & \lambda^{2}+|0\rangle\left\langle\nu_{e}\right|
\end{array}\right),
\end{aligned}
$$

so

$$
\mathrm{P}_{1}[z-\mathrm{K}]^{-1} \mathrm{P}_{1}=z \mathrm{P}_{1}\left[z^{2}-\mathrm{H}_{e}\right]^{-1} \mathrm{P}_{1} .
$$

According to the Feshbach projection formula with $A$ an operator and $P=1-Q$ a projector,

$$
A^{-1}=[Q A Q]^{-1} Q+\left\{P-[Q A Q]^{-1} Q A P \mathcal{G}_{P}\left\{P-P A Q[Q A Q]^{-1}\right\},\right.
$$




$$
\begin{array}{cc}
P A^{-1} P=\mathcal{G}_{P} P, & \mathcal{G}_{P}=\left[P A P-P A Q(Q A Q)^{-1} Q A P\right]^{-1}, \\
Q A^{-1} Q=\mathcal{G}_{Q} Q, & \mathcal{G}_{Q}=\left[Q A Q-Q A P(P A P)^{-1} P A Q\right]^{-1} .
\end{array}
$$

In our case $P=\mathrm{P}_{1}=1-\mathrm{Q}_{1}=1-\mathrm{P}_{2}-\mathrm{P}_{3}, A=z^{2}-\mathrm{H}_{e}$, and we obtain

$$
\mathrm{P}_{1}\left[z^{2}-\mathrm{H}_{e}\right]^{-1} \mathrm{P}_{1}=\left[z^{2}-\mathrm{h}_{0}-\chi_{e}^{\prime}(\mathbf{x}, 0)-\mathrm{P}_{1} \mathrm{H}_{e} \mathrm{Q}_{1}\left[z^{2}-\mathrm{Q}_{1} \mathrm{H}_{e} \mathrm{Q}_{1}\right]^{-1} \mathrm{Q}_{1} \mathrm{H}_{e} \mathrm{P}_{1}\right]^{-1} \mathrm{P}_{1} \text {. }
$$

Here

$$
\begin{aligned}
\mathrm{P}_{1} \mathrm{H}_{e} \mathrm{Q}_{1}\left[z^{2}-\mathrm{Q}_{1} \mathrm{H}_{e} \mathrm{Q}_{1}\right]^{-1} \mathrm{Q}_{1} \mathrm{H}_{e} \mathrm{P}_{1}= & \mathrm{P}_{1} \mathrm{H}_{e} \mathrm{P}_{2}\left[z^{2}-\mathrm{P}_{2} \mathrm{H}_{e} \mathrm{P}_{2}\right]^{-1} \mathrm{P}_{2} \mathrm{H}_{e} \mathrm{P}_{1} \\
& +\mathrm{P}_{1} \mathrm{H}_{e} \mathrm{P}_{3}\left[z^{2}-\mathrm{P}_{3} \mathrm{H}_{e} \mathrm{P}_{3}\right]^{-1} \mathrm{P}_{3} \mathrm{H}_{e} \mathrm{P}_{1} \\
= & \left\langle\nu_{e}\left|\lambda^{2}\left[z^{2}-\lambda^{2}\right]^{-1}\right| 0\right\rangle-\boldsymbol{\epsilon} \cdot \mathbf{p}\left\langle\nu_{m}\left|\left[z^{2}-\lambda^{2}-|0\rangle\left\langle\nu_{m}\right|\right]^{-1}\right| 0\right\rangle \cdot \boldsymbol{\epsilon} \cdot \mathbf{p}
\end{aligned}
$$

and

$$
\begin{gathered}
\left\langle\nu_{e}\left|\lambda^{2}\left[z^{2}-\lambda^{2}\right]^{-1}\right| 0\right\rangle=-\chi_{e}^{\prime}(\mathbf{x}, 0)-z^{2} \hat{\chi}_{e}(\mathbf{x}, z), \\
\left\langle\nu_{m}\left|\left[z^{2}-\lambda^{2}-|0\rangle\left\langle\nu_{m}\right|\right]^{-1}\right| 0\right\rangle=-1+\left[1-\left\langle\nu_{m}\left|\left[z^{2}-\lambda^{2}\right]^{-1}\right| 0\right\rangle\right]^{-1}=-1+\mu(\mathbf{x}, z)^{-1},
\end{gathered}
$$

leading to

$$
\mathrm{P}_{1}\left[z^{2}-\mathrm{H}_{e}\right]^{-1} \mathrm{P}_{1}=\left[z^{2} \boldsymbol{\varepsilon}(\mathbf{x}, z)+(\boldsymbol{\epsilon} \cdot \mathbf{p}) \mu(\mathbf{x}, z)^{-1} \cdot(\boldsymbol{\epsilon} \cdot \mathbf{p})\right]^{-1} \mathrm{P}_{1}=\mathrm{R}_{e}(z) \mathrm{P}_{1}
$$

so

$$
\mathrm{P}_{1}[z-\mathrm{K}]^{-1} \mathrm{P}_{1}=z \mathrm{R}^{e}(z) \mathrm{P}_{1}
$$

Similarly

$$
\mathrm{P}_{4}[z-\mathrm{K}]^{-1} \mathrm{P}_{4}=z \mathrm{R}^{m}(z) \mathrm{P}_{4}
$$

\section{APPENDIX C: DECOMPOSITION OF GREEN'S FUNCTIONS FOR THE LAYERED CASE}

We express $\mathbf{R}_{\boldsymbol{\kappa}}^{e, m}(z)$ in terms of the inverses of scalar operators. Using the Feshbach formula, Eq. (B3), with $A=\mathrm{L}_{\boldsymbol{\kappa}}^{e, m}(z)$ and

$$
\begin{gathered}
\mathrm{P}_{s}=\mathbf{e}_{3} \times \mathbf{e}_{\boldsymbol{\kappa}} \mathbf{e}_{3} \times \mathbf{e}_{\boldsymbol{\kappa}}, \\
\mathrm{Q}_{s}=\mathrm{U}-\mathrm{P}_{s}=\mathbf{e}_{\boldsymbol{\kappa}} \mathbf{e}_{\boldsymbol{\kappa}}+\mathbf{e}_{3} \mathbf{e}_{3},
\end{gathered}
$$

we find, noting that

$$
\begin{gathered}
\left(\mathbf{e}_{3} \times \mathbf{e}_{\boldsymbol{\kappa}}\right) \cdot(\boldsymbol{\epsilon} \cdot \mathbf{p})=\kappa \mathbf{e}_{3}-p \mathbf{e}_{\boldsymbol{\kappa}}, \\
(\boldsymbol{\epsilon} \cdot \mathbf{p}) \cdot\left(\mathbf{e}_{3} \times \mathbf{e}_{\boldsymbol{\kappa}}\right)=-\kappa \mathbf{e}_{3}+p \mathbf{e}_{\boldsymbol{\kappa}} \\
\boldsymbol{\epsilon} \cdot \mathbf{p} \cdot \mathbf{Q}_{s}=(\boldsymbol{\epsilon} \cdot \mathbf{p}) \cdot\left(\mathbf{e}_{\boldsymbol{\kappa}} \mathbf{e}_{\boldsymbol{\kappa}}+\mathbf{e}_{3} \mathbf{e}_{3}\right)=\left(\mathbf{e}_{3} \times \mathbf{e}_{\boldsymbol{\kappa}}\right)\left(\kappa \mathbf{e}_{3}-p \mathbf{e}_{\boldsymbol{\kappa}}\right),
\end{gathered}
$$

etc., that (we skip the subscript $\boldsymbol{\kappa}$ for brevity)

$$
\mathrm{P}_{s} \cdot \mathrm{L}^{e} \cdot \mathrm{P}_{s}=\left[\frac{\zeta^{2}}{\mu}-p \frac{1}{\mu} p\right] \mathrm{P}_{s},
$$




$$
\begin{gathered}
\mathrm{P}_{s} \cdot \mathrm{L}^{e} \cdot \mathbf{Q}_{s}=0, \\
\mathbf{Q}_{s} \cdot \mathrm{L}^{e}(z) \cdot \mathbf{Q}_{s}=\mathrm{L}_{p}^{e}=\left[z^{2} \varepsilon(x, z) \mathrm{U}_{Q}-\left(p \mathbf{e}_{\boldsymbol{\kappa}}-\kappa \mathbf{e}_{3}\right) \frac{1}{\mu(x, z)}\left(p \mathbf{e}_{\boldsymbol{\kappa}}-\kappa \mathbf{e}_{3}\right)\right] \cdot \mathbf{Q}_{s}
\end{gathered}
$$

and similar for $\mathrm{L}_{\boldsymbol{\kappa}}^{m}$ (interchange $\varepsilon$ and $\mu$ ), where

$$
\zeta(x, \kappa, z)^{2}=z^{2} \varepsilon(x, z) \mu(x, z)-\kappa^{2} .
$$

Thus,

$$
\mathbf{R}^{e, m}(z)=\mathbf{R}_{s}^{e, m}(z)+\mathbf{R}_{p}^{e, m}(z),
$$

where, with

$$
\begin{gathered}
R_{s}^{e}(z)=\left[z^{2} \varepsilon-\frac{\kappa^{2}}{\mu}-p \frac{1}{\mu} p\right]^{-1}, \quad R_{s}^{m}(z)=\left[\frac{\zeta^{2}}{\varepsilon}-p \frac{1}{\varepsilon} p\right]^{-1}, \\
\mathrm{R}_{s}^{e}(z)=R_{s}^{e}(z) \mathrm{P}_{s}, \quad \mathrm{R}_{s}^{m}(z)=R_{s}^{m}(z) \mathrm{P}_{s}, \\
\mathrm{R}_{p}^{e}(z)=\left[z^{2} \varepsilon \mathrm{U}_{Q}-\left(p \mathbf{e}_{\boldsymbol{\kappa}}-\kappa \mathbf{e}_{3}\right) \frac{1}{\mu}\left(p \mathbf{e}_{\boldsymbol{\kappa}}-\kappa \mathbf{e}_{3}\right)\right]^{-1} \mathrm{Q}_{s}, \\
\mathrm{R}_{p}^{m}(z)=\left[z^{2} \mu \mathrm{U}_{Q}-\left(p \mathbf{e}_{\boldsymbol{\kappa}}-\kappa \mathbf{e}_{3}\right) \frac{1}{\varepsilon}\left(p \mathbf{e}_{\boldsymbol{\kappa}}-\kappa \mathbf{e}_{3}\right)\right]^{-1} \mathrm{Q}_{s} .
\end{gathered}
$$

Now let

$$
\mathrm{R}_{p}^{e}(z)=A \mathbf{e}_{\boldsymbol{\kappa}} \mathbf{e}_{\boldsymbol{\kappa}}+B \mathbf{e}_{\boldsymbol{\kappa}} \mathbf{e}_{3}+C \mathbf{e}_{3} \mathbf{e}_{\boldsymbol{\kappa}}+D \mathbf{e}_{3} \mathbf{e}_{3} .
$$

Since

$$
\mathrm{L}_{p}^{e} \cdot \mathrm{R}_{p}^{e}=\mathrm{Q}_{s}=\mathbf{e}_{\boldsymbol{\kappa}} \mathbf{e}_{\boldsymbol{\kappa}}+\mathbf{e}_{3} \mathbf{e}_{3},
$$

we find by comparing coefficients that

$$
\begin{gathered}
\left(z^{2} \varepsilon-p \frac{1}{\mu} p\right) A+p \frac{\kappa}{\mu} C=1, \\
\frac{\zeta^{2}}{\mu} D+\frac{\kappa}{\mu} p B=1, \\
\frac{\kappa}{\mu} p A+\frac{\zeta^{2}}{\mu} C=0, \\
\left(z^{2} \varepsilon-p \frac{1}{\mu} p\right) B+p \frac{\kappa}{\mu} D=0 .
\end{gathered}
$$

Let

$$
R_{p}^{e}(z)=\left[z^{2} \varepsilon-p \frac{z^{2} \varepsilon}{\zeta^{2}} p\right]^{-1}, \quad R_{p}^{m}(z)=\left[z^{2} \mu-p \frac{z^{2} \mu}{\zeta^{2}} p\right]^{-1} .
$$

Then 


$$
\begin{gathered}
A=R_{p}^{e}(z), \quad B=-R_{p}^{e}(z) p \frac{\kappa}{\zeta^{2}}, \quad C=-\frac{\kappa}{\zeta^{2}} p R_{p}^{e}(z), \\
D=\frac{\mu}{\zeta^{2}}+\frac{\kappa}{\zeta^{2}} p\left[z^{2} \varepsilon-p \frac{z^{2} \varepsilon}{\zeta^{2}} p\right] p \frac{\kappa}{\zeta^{2}},
\end{gathered}
$$

with similar results for the magnetic case. Hence

$$
\begin{aligned}
& \mathrm{R}^{e}(z)=R_{s}^{e}(z) \mathbf{e}_{3} \times \mathbf{e}_{\boldsymbol{\kappa}} \mathbf{e}_{3} \times \mathbf{e}_{\boldsymbol{\kappa}}+\left(\mathbf{e}_{\boldsymbol{\kappa}}-\frac{\kappa}{\zeta^{2}} p \mathbf{e}_{3}\right) R_{p}^{e}(z)\left(\mathbf{e}_{\boldsymbol{\kappa}}-p \frac{\kappa}{\zeta^{2}} \mathbf{e}_{3}\right)+\frac{\mu}{\zeta^{2}} \mathbf{e}_{3} \mathbf{e}_{3}, \\
& \mathrm{R}^{m}(z)=R_{s}^{m}(z) \mathbf{e}_{3} \times \mathbf{e}_{\boldsymbol{\kappa}} \mathbf{e}_{3} \times \mathbf{e}_{\boldsymbol{\kappa}}+\left(\mathbf{e}_{\boldsymbol{\kappa}}-\frac{\kappa}{\zeta^{2}} p \mathbf{e}_{3}\right) R_{p}^{m}(z)\left(\mathbf{e}_{\boldsymbol{\kappa}}-p \frac{\kappa}{\zeta^{2}} \mathbf{e}_{3}\right)+\frac{\varepsilon}{\zeta^{2}} \mathbf{e}_{3} \mathbf{e}_{3} .
\end{aligned}
$$

Note that

$$
R_{p}^{e}(z)=\frac{1}{p} \frac{\zeta^{2}}{z^{2} \varepsilon} R_{s}^{m}(z) p \frac{1}{\varepsilon},
$$

so $\mathrm{R}_{p}^{e}(z)$ can be expressed in terms of the scalar magnetic s-polarized Green's function but the vectors in front and behind are quite different. From the above expressions the corresponding Green's functions, introduced in Sec. V, now follow.

${ }^{1}$ V. G. Veselago, Sov. Phys. Usp. 10, 509 (1968).

${ }^{2}$ J. B. Pendry, Phys. Rev. Lett. 85, 3966 (2000).

${ }^{3}$ V. Veselago, L. Braginsky, V. Shklover, and C. Hafner, J. Comput. Theor. Nanosci. 3, 1 (2006).

${ }^{4}$ G. W. 't Hooft, Phys. Rev. Lett. 87, 249701 (2001); P. M. Valanju and R. M. Walser, ibid. 88, 187401 (2002); N. Garcia and M. Nieto-Garcia, ibid. 88, 207403 (2002); M. Nieto-Vesperinas, J. Opt. Soc. Am. A 21, 491 (2004); D. Maystre and S. Enoch, ibid. 21, 122 (2004); I. Stockman, Phys. Rev. Lett. 98, 177404 (2007).

${ }^{5}$ D. R. Smith, W. J. Padilla, D. C. Vier, S.C. Nemat-Nasser, and S. Schultz, Phys. Rev. Lett. 84, 4184 (2000); R. A. Shelby, D. R. Smith, S. C. Nemat-Nasser, and S. Schultz, Appl. Phys. Lett. 78, 489 (2001); see also N. Liu, H. Guo, L. Fu, S. Kaiser, H. Schweizer, and H. Giessen, Nature Mater. 7, 31 (2008) and references quoted.

${ }^{6}$ A. Tip, Phys. Rev. A 57, 4818 (1998).

${ }^{7}$ A. Tip, Phys. Rev. E 69, 016610 (2004).

${ }^{8}$ J. D. Jackson, Classical Electrodynamics (Wiley, New York, 1962).

${ }^{9}$ H. T. Dung, S. Y. Buhman, D.-G. Welsch, S. Scheel, and J. Kästel, Phys. Rev. A 68, 043816 (2003).

${ }^{10}$ S. Guenneau, B. Gralak, and J. B. Pendry, Opt. Lett. 30, 1204 (2005).

${ }^{11}$ C. Raabe, S. Scheel, and D.-G. Welsch, Phys. Rev. A 75, 053813 (2007).

${ }^{12}$ R. E. Collin, PIER B 19, 233 (2010).

${ }^{13}$ T. G. Mackay and A. Lakhtakia, Phys. Rev. Lett. 99, 189701 (2007); M. I. Stockman, ibid. 99, 189702 (2007).

${ }^{14}$ J. B. Pendry, D. Schurig, and D. R. Smith, Science 312, 1780 (2006); D. Schurig, J. B. Pendry, and D. R. Smith, Opt. Express 14, 9794 (2006; U. Leonhardt and T. G. Philbin, New J. Phys. 8, 247 (2006). 